\title{
3-D chamfer distances and norms in anisotropic grids
}

\author{
Céline Fouard ${ }^{\mathrm{a}, \mathrm{b}, \mathrm{c}, *}$, Grégoire Malandain $^{\mathrm{a}}$ \\ ${ }^{a}$ Epidaure Research Project, INRIA, 2004 route des Lucioles, BP 92, 06902 Sophia-Antipolis cedex, France \\ ${ }^{\mathrm{b}}$ TGS Europe SA, PA Kennedy 1, BP 227, F-33708 Mérignac cedex, France \\ ${ }^{\mathrm{c}}$ INSERM U455 Toulouse, France
}

Received 16 January 2004; received in revised form 5 May 2004; accepted 29 June 2004

\begin{abstract}
Chamfer distances are widely used in image analysis and many authors have investigated the computation of optimal chamfer mask coefficients. Unfortunately, these methods are not systematized: calculations have to be conducted manually for every mask size or image anisotropy. Since image acquisition (e.g. medical imaging) can lead to discrete anisotropic grids with unpredictable anisotropy value, automated calculation of chamfer mask coefficients becomes mandatory for efficient distance map computations. This article presents an automatic construction for chamfer masks of arbitrary sizes. This allows, first, to derive analytically the relative error with respect to the Euclidean distance, in any 3-D anisotropic lattice, and second, to compute optimal chamfer coefficients. In addition, the resulting chamfer map verifies discrete norm conditions.
\end{abstract}

(C) 2004 Elsevier B.V. All rights reserved.

Keywords: Chamfer distance; Anisotropic lattice; Farey triangulation

\section{Introduction}

Distance transformations (DTs) turn binary images into grey-level images where the value of each foreground pixel corresponds to its shortest distance to the background. They are widely used in image analysis since they allow recovering morphometric features of a binary shape. Among other applications, they can be used to compute skeletons, medial axis [1], or Voronoï diagrams or assist in shape-based interpolation [2].

The primary motivation of this work is the computation of statistically significant morphometric parameters of the cerebral microvasculature (the micro-vessels comprise capillaries, small veins and arteries of the cerebral vasculature). This information could help understand and/or simulate functional imaging modalities (e.g. TEP or fMRI) [3]. While the smallest of the micro-vessels have a diameter

\footnotetext{
* Corresponding author. Address: Epidaure Research Project, INRIA, 2004 route des Lucioles, BP 92, 06902 Sophia-Antipolis cedex, France.

E-mail addresses: celine.fouard@sophia.inria.fr (C. Fouard), http://www-sop.inria.fr/epidaure/personnel/celine.fouard/ (C. Fouard), gregoire.malandain@sophia.inria.fr (G. Malandain).
}

of a few micrometers, we want to process large areas (a few millimeters) in cerebral histological slices (see Ref. [4] for details about the used material). This results in a huge amount of data. The imaged portion of cerebral slice shown in Fig. 1 was typically acquired with a confocal microscope as a mosaic of $18512 \times 512 \times 1283$-D images, with voxel size $1.22 \times 1.22 \times 3 \mu \mathrm{m}^{3}$. This corresponds to a virtual image of approximately $3400 \times 1400 \times 128$ voxels. ${ }^{1}$ Distance computation is used on these mosaics to extract the center lines of the vessels and to compute their radii [5].

Clearly this volume of data cannot be entirely loaded (and processed) in the central memory of standard computers. This makes the adaptation of Euclidean distance transform (EDT) algorithms [6] less attractive than chamfer distance computation that can be easily adapted to mosaics. Optimal chamfer coefficients for isotropic images can be found in the literature [7]. Resampling the anisotropic mosaic presented in Fig. 1 into an isotropic lattice will approximately double the volume of input data (here from 450 to $900 \mathrm{Mb}$ ). While modern computer disk capacity is

\footnotetext{
${ }^{1}$ Overlaps of about 50 voxels exist between adjacent images for automatic and precise realignments of all images to form a mosaic [5].
} 

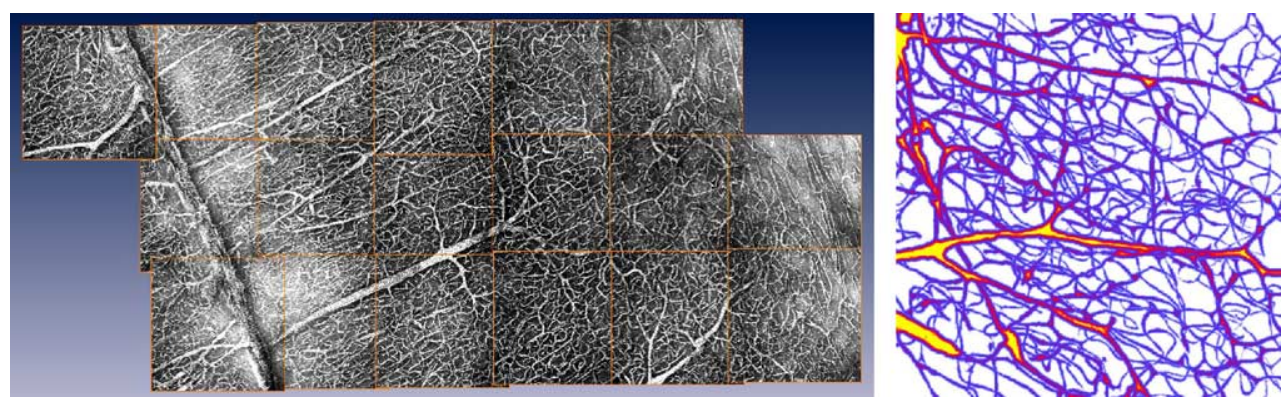

Fig. 1. Portion (maximum intensity projection) of a cerebral histological slice, acquired as an image mosaic (corresponding to an area of $4 \times 1.8 \mathrm{~mm}^{2}$ ) and a detail of distance map on this mosaic.

sufficient to store typically a few anisotropic mosaics together with the associated results, they cannot accommodate as many in the isotropic case (a typical acquisition consists in a mosaic of more than 100 images, covering an area between 25 and $100 \mathrm{~mm}^{2}$ ).

The computation of optimal chamfer coefficients for anisotropic images has also been discussed in the literature [8], but here again in a non-automated fashion. Inevitable variations of the anisotropy factor (the ratio between the inter-slice distance and the pixel size) between acquisitions prompted us to develop a fully automated algorithm to compute optimal chamfer coefficients in anisotropic lattices.

The remainder of this article is organized as follows. In Section 2, we recall some properties of chamfer distances and chamfer mask, the notion of mask convexity is introduced, and the computation of optimal chamfer coefficients, as presented in the literature, is discussed. The analytical derivation of the relative error between chamfer and Euclidean distances is detailed in Section 3 while Section 4 presents the norm conditions. Section 5 describes the automatic construction of a chamfer mask and the computation of sets of optimal chamfer coefficients. Some results are provided in Section 6.

\section{Recalls on chamfer distance}

In this section, we recall the properties of chamfer masks, as proposed in Ref. [9] and detailed in Ref. [10]. We then describe classical ways to compute optimal chamfer mask coefficients.

We denote $\mathbb{R}$ the field of real numbers, and $\mathbb{Z}$ the ring of integers. A vector space is defined with a commutative field $K$. Given $n \in \mathbb{N}^{*}$, then $K^{n}$ is a vector space on $K$. For example $\mathbb{R}^{n}$ is a vector space on $\mathbb{R}$. However, since $\mathbb{Z}$ is a commutative ring and not a field, $\mathbb{Z}^{n}$ does not define a vectorial space on $\mathbb{Z}$.

Distances and norms are usually defined on the finite space $\mathbb{R}^{n}$ and takes real values. In discrete geometry, however, we sometimes need to have discrete distances defined on $\mathbb{Z}^{n}$ with their values in $\mathbb{Z}$. Since $\mathbb{Z}^{n}$ is not a vector space, the notion of distances and norms have to be extended.

In the following, given a subgroup $F$ of $\mathbb{R}$, we denote by $p=\left(p_{i}\right)_{i=1, \ldots, n}$ an element of $F^{n}$ where $\forall i \in[1, n], p_{i} \in F$. A vector $\mathbf{v}$ will also be denoted by $\left(v_{i}\right)_{i=1, \ldots, n}$.

\subsection{Distance and norm}

Definition 2.1 (Distance) Let $E$ be a non-empty set (e.g. $\mathbb{Z}^{n}$ ), and $F$ a subgroup of $\mathbb{R}$. A distance on $E$, taking its value in $F$, called $(d, E, F)$, is an application $d: E \times E \mapsto F$ which satisfies the following properties:

$$
\begin{array}{ccc}
\text { (positivity) } & \forall p, q \in E & d(p, q) \geq 0 \\
\text { (definition) } & \forall p, q \in E & d(p, q)=0 \Leftrightarrow p=q \\
\text { (symmetry) } & \forall p, q \in E & d(p, q)=d(q, p)
\end{array}
$$

(triangularinequality) $\forall p, q, r \in E d(p, q) \leq d(p, r)+d(r, q)$

$d_{1}, d_{2}$ (the Euclidean distance, also denoted $d_{\mathrm{E}}$ ), and $d_{\infty}$, defined as follows, are usual distances in $\mathbb{R}^{n}$ :

$$
\begin{aligned}
& d_{1}(p, q)=\sum_{i=1}^{n}\left|q_{i}-p_{i}\right|, \quad d_{2}(p, q)=\sqrt{\sum_{i=1}^{n}\left|q_{i}-p_{i}\right|^{2}}, \\
& d_{\infty}(p, q)=\max _{i=1, \ldots, n}\left|q_{i}-p_{i}\right|
\end{aligned}
$$

A discrete distance $d$ is defined as a distance $\left(d, \mathbb{Z}^{n}, \mathbb{Z}\right) . d_{1}$ and $d_{\infty}$ are obviously discrete distances, but not $d_{\mathrm{E}}$. Moreover, neither the square, nor the truncature of $d_{\mathrm{E}}$ are distances (they do not satisfy triangular inequality). Unfortunately, $d_{\mathrm{E}}$ is the most commonly used continuous distance, because of its rotation invariance. Chamfer distances offer a way to approximate a proportional estimation of the Euclidean distance in the discrete metric space $\left(\mathbb{Z}^{n}, \mathbb{Z}\right)$.

A norm is usually defined on a vector space. The notion of module allows to generalize the notion of vector space to sets as $\mathbb{Z}^{n}$. A module is abstractly very similar to a vector space although, in modules, coefficients are taken in rings (such $\mathbb{Z}$ ). 
Definition 2.2 (Module) Let $A$ be a commutative ring (e.g. $\mathbb{Z})$, with identity elements noted 0 and 1 . A set $E$ (e.g. $\mathbb{Z}^{n}$ ) defines a module on $A$ (or A-module) called $(E, A)$, if $E$ owns a commutative group operation ( + ), an external law $(\cdot)$ and satisfies the following properties:

$$
\text { (identity) }
$$

(associativity)

(distributivity of scalar sums)

(distributivity of vector sums)

$$
\begin{gathered}
\forall \mathbf{x} \in E \\
\forall \mathbf{x} \in E, \forall \lambda, \mu \in A, \\
\forall \mathbf{x} \in E, \forall \lambda, \mu \in A, \\
\forall \mathbf{x}, \mathbf{y} \in E, \forall \lambda \in A,
\end{gathered}
$$

The main difference between a module and a vector space is the non-invertibility (with respect to the external law) of the elements of its associated ring (e.g. $2 \in \mathbb{Z}$, but $\frac{1}{2} \notin \mathbb{Z}$ ). A base of a module of dimension $n$ is a family of $n$ independent vectors $\left(\mathbf{v}_{i}\right)_{i=1, \ldots, n}$, i.e.

$\forall \alpha \in A^{n}, \quad \sum_{i=1}^{n} \alpha_{i} \mathbf{v}_{i}=0, \quad \Leftrightarrow \forall i \in[1, \ldots, n] \alpha_{i}=0$.

But, a linearly independent family of $n$ vectors may not be a base of $A^{n}$. We define a norm in a module in the same way as in a vector space.

Definition 2.3 (Norm) Let $(E, A)$ be a module, and $F$ be a subgroup of $\mathbb{R}$. A norm on $(E, A)$ taking its value in $F$ is an application $g: E \mapsto F$ which satisfies:

$$
\begin{array}{ccc}
\text { (positivity) } & \forall \mathbf{x} \in E & g(\mathbf{x}) \geq 0 \\
\text { (definition) } & \forall \mathbf{x} \in E & g(\mathbf{x})=0 \Leftrightarrow \mathbf{x}=0 \\
\text { (triangular inequality) } & \forall \mathbf{x}, \mathbf{y} \in E & g(\mathbf{x}+\mathbf{y}) \leq g(\mathbf{x})+g(\mathbf{y}) \\
\text { (homogeneity) } & \forall \mathbf{x} \in E, \forall \lambda \in A, & g(\lambda \mathbf{x})=|\lambda| g(\mathbf{x})
\end{array}
$$

\subsection{Distance maps and distance transformations}

We now consider an image $I$ as an application from a finite set $E$ of $\mathbb{Z}^{n}$ to $\mathbb{Z}$.

Definition 2.4 (Distance map) Given a binary image $I$, let $X=\{p \in E \mid I(p)=1\}$ be the foreground and $\bar{X}=\{p \in$ $E \mid I(p)=0\}$ be the background. The distance map of $I$ is a grey level image where the value of each point of the foreground corresponds to its shortest distance to the background, i.e.

$$
\mathrm{DM}_{X}:\left\{\begin{array}{l}
E \rightarrow \mathbb{N} \\
p \mapsto d(p, \bar{X})=\inf _{q \in \bar{X}} d(p, q)
\end{array}\right.
$$

Exact Euclidean maps can be computed through EDT. Several EDT have been proposed, using morphological operators [11,12], filters [13], several path on rows and columns [14], propagating vectors $[15,16]$, or Voronoï diagrams [17]. A comprehensive review of distance transformations can be found in Ref. [6]. Practical implementations lead to time and/or memory consuming algorithms: this makes them not suitable for our aimed application that requires extremely simple, fast, and low memory consuming tools. Chamfer maps, first proposed by Montanari [18] and popularized by Borgefors [19], are then an adequate choice because of their generality, $\cdot \mathbf{x}=\mathbf{x}$

$$
\cdot(\mu \cdot \mathbf{x})=(\lambda \mu) \cdot \mathbf{x}
$$

$=\lambda \cdot \mathbf{x}+\mu \cdot \mathbf{x}$

simplicity and efficiency. Indeed, they can be efficiently computed with a two-scan algorithm [20] and propagation of local distances through chamfer masks.

\subsection{Chamfer masks and chamfer distances}

In this section we define more precisely chamfer masks and chamfer distances definition on a module. Let $(E, A)$ be a module and $F$ be a subgroup of $\mathbb{R}$.

Definition 2.5 (Chamfer mask) A chamfer mask is a finite set $m_{\mathrm{C}}=\left\{\left(\mathbf{v}_{k}, \omega_{k}\right) \in E \times F, 1 \leq k \leq m\right\}$ which contains at least a base of $E$, and which satisfies the following properties:

(positive weights) $\forall k \omega_{k}>0$ and $\mathbf{v}_{k} \neq \mathbf{0}$

(central symmetry) $\forall(\mathbf{v}, \omega) \in m_{\mathrm{C}} \Rightarrow\left(\left( \pm v_{i}\right), \omega\right) \in m_{\mathrm{C}}$

with $\mathbf{v}=\left(v_{i}\right)$

We call $C(E, A, F)$ the set of chamfer masks in the module $(E, A)$ with their coefficients in $F$.

Chamfer mask vectors $\mathbf{v}_{k}$ represent legal displacements in the neighborhood of the central point. Several neighborhood sizes can be considered. The mask size is defined as the number of voxels (included the central one) in this neighborhood.

A point $p \in \mathbb{Z}^{n}$ is called visible (from the origin $O$ ) if there is no point of $\mathbb{Z}^{n}$ located on the line $(O p)$ between $O$ and $p$.

Non-visible points are usually suppressed from the chamfer masks. Indeed, consider a non-visible point $p$. By definition, there exist a point $q$ which verifies $\overrightarrow{O p}=\lambda \vec{O} q$ with $\lambda \in \mathbb{Z}^{+}$. On one hand, if $\omega_{\vec{O} p}=\lambda d_{\mathrm{c}}(O, q)$, the mask is redundant since there are two ways to compute $d_{\mathrm{c}}(O, p)$ (either directly with $\omega_{\vec{O} p}$ by propagation from $q$ ). On the other hand, if $\omega_{\overrightarrow{O p}} \neq \lambda d_{\mathrm{c}}(O, q)$, the distance map is no more homogeneous along $\overrightarrow{O p}$ direction.

The suppression of non-visible points from chamfer masks allows not only to avoid these problems but also to simplify chamfer coefficients computation. Fig. 2 shows two examples of 3 -D $3 \times 3 \times 3$ chamfer mask geometry containing only visible points. 


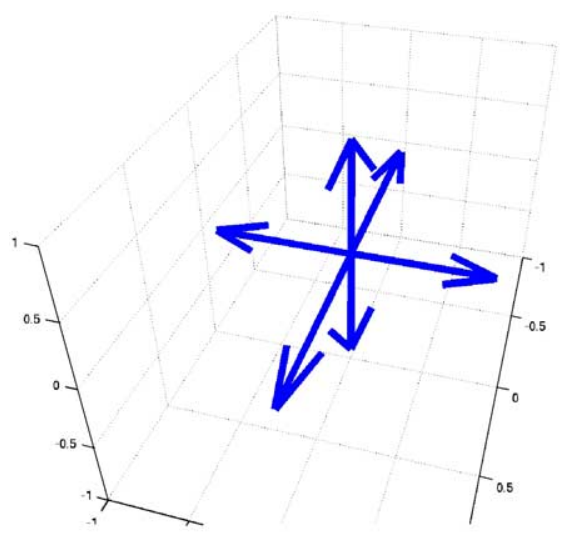

(a)

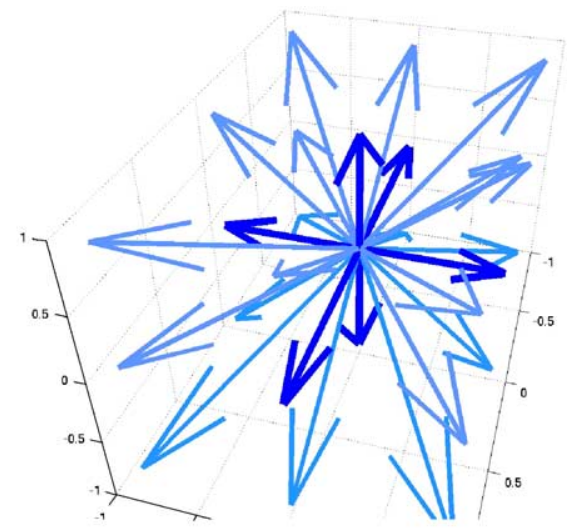

(b)

Fig. 2. Examples of 3-D $3 \times 3 \times 3$ chamfer mask geometries.

Definition 2.6 (Path from $p$ to $q$ ) Given a chamfer mask $m_{\mathrm{C}}$ and two points $p, q \in E$, a path from $p$ to $q$ is a sequence of vectors $\mathbf{v}_{k} \in m_{\mathrm{C}}$ going from $p$ to $q$. We obtain:

$\overrightarrow{p q}=\sum_{\mathbf{v}_{k} \in m_{\mathrm{C}}} n_{k} \cdot \mathbf{v}_{k}$

The cost $W$ of the path $\mathscr{P}_{p q}$ is defined by:

$W\left(\mathscr{P}_{p q}\right)=\sum_{k=1}^{m} n_{k} \cdot \omega_{k}$

Since a mask $m_{\mathrm{C}}$ contains a base of $E$, such a path $\mathscr{P}_{p q}$ always exists for any couple of points $(p, q)$.

Definition 2.7 (Chamfer distance) A chamfer distance $d_{\mathrm{C}}$ between two points $p$ and $q$ in $E$ is the minimum of the costs associated to paths $\mathscr{P}_{p q}$ linking $p$ to $q$ :

$d_{\mathrm{C}}=\min _{\mathscr{P}_{p q}} W\left(\mathscr{P}_{p q}\right)$

\subsection{Distance and norm properties of a chamfer mask}

Given a module $(E, A)$ and a chamfer mask, the following propositions hold.

Proposition 2.1. Let $(E, A)$ be a module, and $F$ be a subgroup of $\mathbb{R}$. Given a chamfer mask $m_{\mathrm{C}} \in C(E, A, F)$, then the chamfer distance $d_{\mathrm{C}}$ associated to this mask is a distance on $E$.

The proof can be found in Refs. [21,22]. Moreover, Thiel proved in Ref. [10] that a chamfer distance is a norm on a vector space.

Proposition 2.2. Let $K$ be a commutative field, E be a vector space on $K$ and $F$ be a subgroup of $\mathbb{R}$. Given a chamfer mask $m_{\mathrm{C}} \in \mathcal{C}(E, K, F)$, then $d_{m}$ induces a norm on $(E, K)$.

A chamfer distance induces a norm on a vector space. However, $\mathbb{Z}^{n}$ is not a vector space but a module (because of the non-invertibility of the external law). Thus, for a chamfer distance to be a norm on a module, additional constraints have to be verified. To this end, Remy and Thiel [9] introduced the notion of equivalent rational ball.

Definition 2.8 (Equivalent rational mask) The equivalent rational mask $m_{\mathrm{C}}^{\prime}$ of a chamfer mask $m_{\mathrm{C}}=\left\{\left(\mathbf{v}_{k}, \omega_{k}\right)\right.$, $1 \leq k \leq m\}$, is defined by:

$m_{\mathrm{C}}^{\prime}=\left\{\left(\frac{x_{k}}{\omega_{k}}, \frac{y_{k}}{\omega_{k}}, \frac{z_{k}}{\omega_{k}}, \cdots\right) \in \mathbb{Q}^{n}:\left(\left(x_{k}, y_{k}, z_{k}, \ldots\right), \omega_{k}\right) \in m_{\mathrm{C}}\right\}$

Each point of the equivalent rational mask is obtained by dividing the vector $\mathbf{v}_{k} \in m_{\mathrm{C}}$ by its associated weight $\omega_{k}$ : it is then approximately located at unit distance (up to a multiplicative constant) from the origin. The polyhedron formed by a triangulation of the equivalent rational mask is called equivalent rational ball (building a triangulation with good properties will be addressed in Section 3.1).

Proposition 2.3. A chamfer mask induces a norm on $\mathbb{Z}^{n}$ if and only if its equivalent rational ball is convex.

Proof of this proposition can be found in Refs. [9,10]. Fig. 3 shows equivalent rational balls of two chamfer masks: (a) induces a norm on $\mathbb{Z}^{3}$, (b) does not.

\subsection{Optimal coefficients calculation}

A chamfer distance between two points $p$ and $q$ computed with a chamfer mask $m_{\mathrm{C}}=\left\{\left(\mathbf{v}_{k}, \omega_{k}\right), 1 \leq k \leq m\right\}$ is a discrete sum of chamfer coefficients: $d_{\mathrm{C}}(p, q)=\sum_{k=1}^{m} n_{k} \omega_{k}$ where $\overrightarrow{p q}=\sum_{k=1}^{m} n_{k} \mathbf{v}_{k}$.

To obtain the best approximation of the Euclidean distance, optimal weights, that yield the best accuracy, have to be calculated. This is a pivotal point for chamfer distances. The first method for this computation was proposed by Borgefors for dimension 2, 3 or more in cubic isotropic grids [7,23]. The maximum accuracy depended on the mask size: to increase precision, the number of $\left(\mathbf{v}_{k}, \omega_{k}\right)$ couples in the chamfer mask had to be increased. 


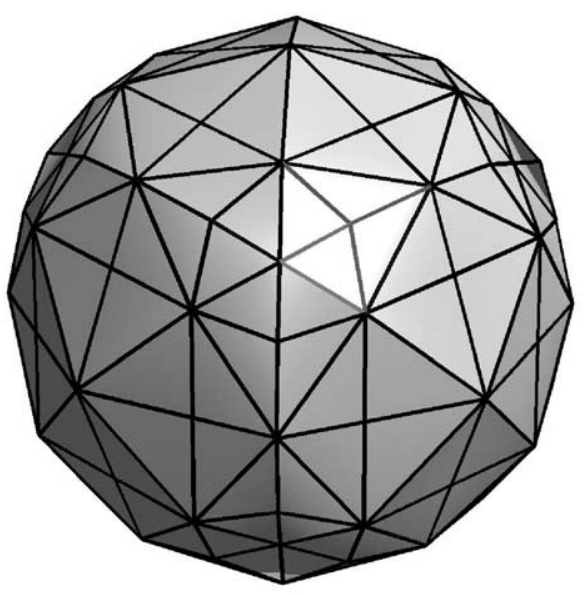

(a) chamfer mask inducing a discrete norm on $Z^{3}$

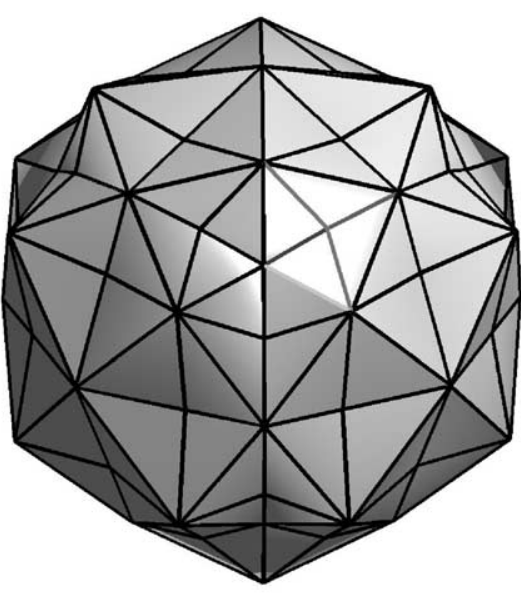

(b) chamfer mask not inducing a discrete norm on $\mathrm{Z}^{3}$

Fig. 3. Examples of $3-\mathrm{D} 5 \times 5 \times 5$ chamfer mask equivalent rational balls.

Some chamfer coefficients calculations are reviewed below.

In Ref. [23], the coefficient computation for a $2-D 3 \times 3$ isotropic chamfer mask is presented. For symmetrical reasons, there are only two coefficients to be calculated: $a$ (the horizontal one), and $b$ (the diagonal one), as shown in Fig. 4 Moreover, only points such that $0 \leq y \leq x$ are considered (other cases are deduced by symmetry). To find optimal coefficients, Borgefors minimizes the maximal error of the chamfer distance with respect to the Euclidean distance. The calculation is performed for the points located on a straight line $x=M$ (Fig. 5). For each point $p(M, y)$ located on the line $x=M$, we get the following quantities:

- the Euclidean distance, $d_{\mathrm{E}}(O, p)=\sqrt{M^{2}+y^{2}}$,

- the chamfer distance, $d_{\mathrm{C}}(O, p)=y b+(M-y) a=y(b-a)+$ $M a$, and

- the absolute error, $E(y)=d_{\mathrm{C}}(y)-d_{\mathrm{E}}(y)=y(b-a)+$ $M a-\sqrt{M^{2}+y^{2}}$

under the conditions $b>a$ and $b<2 a$ to be sure to consider the shortest path.

The derivative of the function $E(y), \mathrm{d} E / \mathrm{d} y=(b-a)-y /$ $\sqrt{M^{2}+y^{2}}$ cancels for $y_{1}=M(b-a) / \sqrt{1-(b-a)^{2}}$. The error is then extremal for three values of $y,\left\{0, y_{1}, M\right\}$,

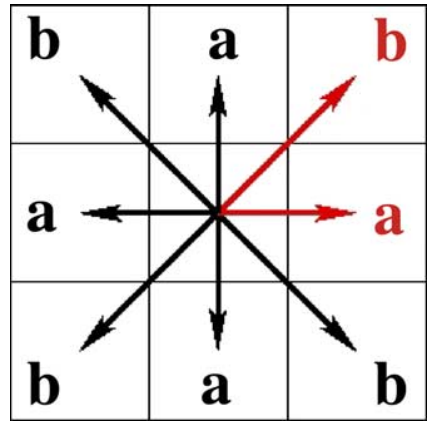

Fig. $4.3 \times 3$ Mask. that corresponds respectively to the following three error values, $E_{0}=(\mathrm{a}-1) M, E_{1}=\left(a-\sqrt{1-(b-a)^{2}}\right) M$, and $E_{2}=(b-\sqrt{2}) M$.

The maximum of these three errors is minimized, yielding optimal real values for $a$ and $b$ :

$a_{\mathrm{opt}}=\frac{1+\sqrt{2 \sqrt{2}-2}}{2} \approx 0.95509 \ldots$

and

$b_{\mathrm{opt}}=\frac{2 \sqrt{2}-1+\sqrt{2 \sqrt{2}-2}}{2} \approx 1.36930 \ldots$

Note that $a_{\mathrm{opt}}<1$ and $b_{\mathrm{opt}}<\sqrt{2}$. Setting $a_{\mathrm{opt}}=1$ and $b=\sqrt{2}$ amounts to considering the error along on the horizontal

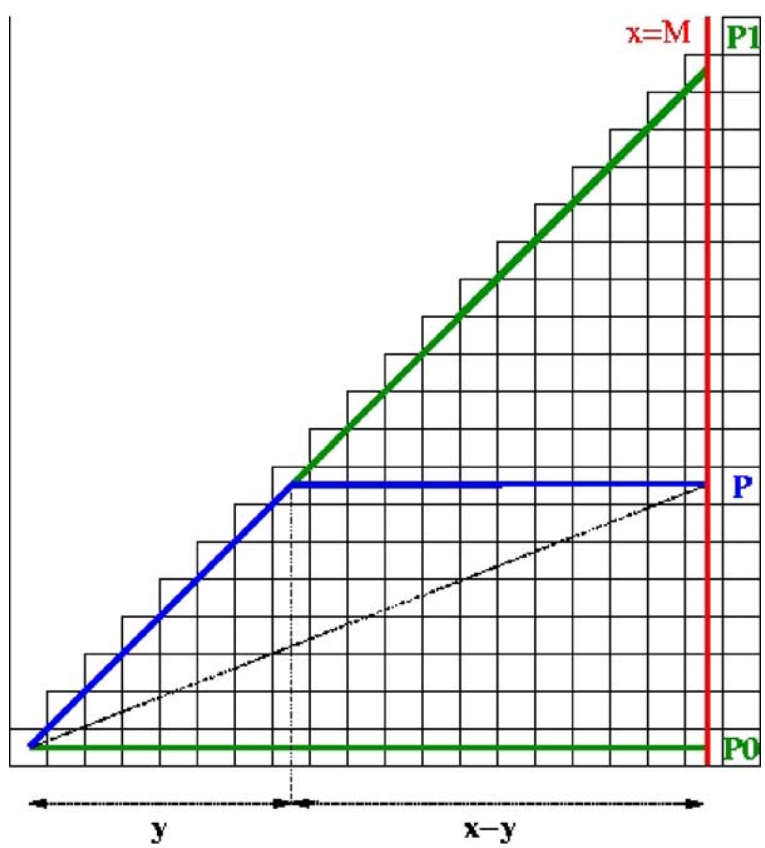

Fig. 5. Coefficients computation a straight line $x=M$. 


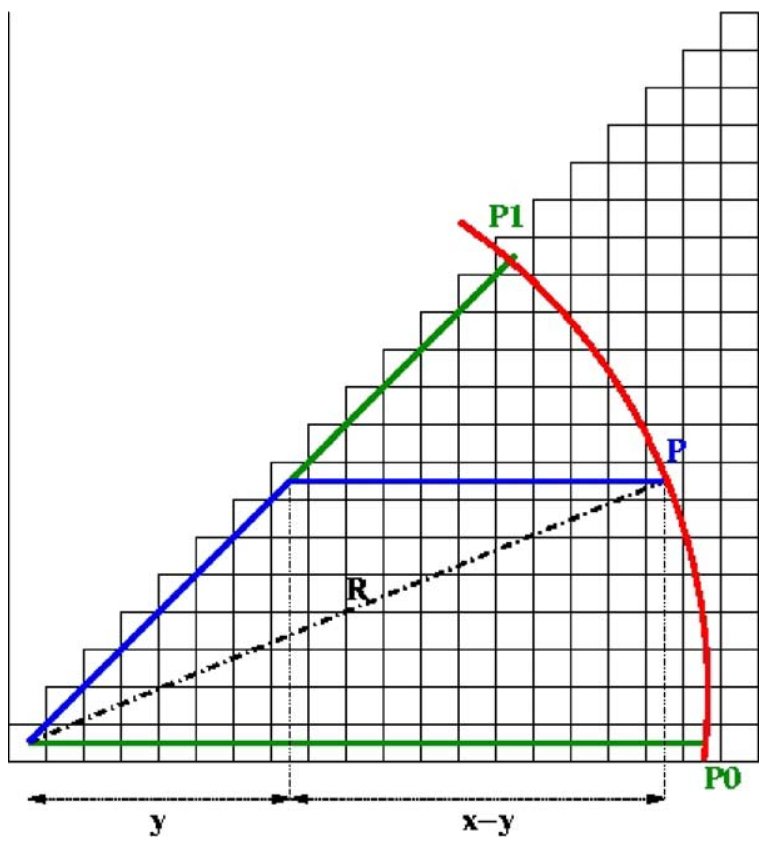

Fig. 6. Coefficients computation on a circle $R=M$.

and diagonal directions only. The maximum error is located on a direction at about 23 degrees of the horizontal direction.

Verwer commented that this approach induces an anisotropy in the error minimization scheme by giving too much importance to the diagonal direction [24]. He proposed an error computation on a unit circle (cf Fig. 6), which is equivalent to computing a relative error on a straight line (see Table 1 for a comparison between both calculation schemes).

In these schemes, the calculation of the optimal coefficients, $a_{\mathrm{opt}}$ and $b_{\mathrm{opt}}$, yields real values, and thus results in a real chamfer distance (defined on the metric space $\left.\left(\mathbb{Z}^{n}, \mathbb{R}\right)\right)$. To get a discrete distance, i.e. defined on $\left(\mathbb{Z}^{n}, \mathbb{Z}\right)$, these optimal real coefficients, $a_{\mathrm{opt}}$ and $b_{\mathrm{opt}}$, have to be approximated by integer coefficients, $a_{\text {int }}$ and $b_{\text {int }}$.

Looking for a chamfer distance that is an approximation of the Euclidean distance up to a real multiplicative constant, say $\varepsilon$, allows a certain freedom in the choice of these integer coefficients. The chamfer distance can be expressed as $d_{\mathrm{C}}=\sum_{k=1}^{m} n_{k} \omega_{k}$ with $\omega_{k} \in \mathbb{Z}$, and is related to the Euclidean distance by $d_{\mathrm{C}} / \varepsilon \approx d_{\mathrm{E}}$. For instance, approximating $b_{\text {opt }} / a_{\text {opt }}$ by $b_{\text {int }} / a_{\text {int }}$ yields the popular coefficients $(3,4)$.

These calculation have been extended to larger masks $[23,24]$ and to higher dimensions [7]. Anisotropic lattices have also been considered $[8,25,26]$. However, those calculations remain tedious, are not systematized and thus have to be conducted manually for every mask size or anisotropy value.

The next section presents the analytical derivation of the relative error that will further allow the automated
Table 1

Summary of different calculation schemes for the $3 \times 3$ chamfer mask

\begin{tabular}{lll}
\hline & Calculation & \\
\cline { 2 - 3 } & On a line $x=M$ & On a circle $R=M$ \\
\hline $\begin{array}{l}\text { Point } \\
\text { coordinates }\end{array}$ & $P(M, y)$ & $P\left(\sqrt{M^{2}-y^{2}}, y\right)$ \\
$\begin{array}{l}\text { Interval } \\
\text { extremities }\end{array}$ & $y_{0}=0, y_{2}=M$ & $y_{0}=0, y_{2}=M / \sqrt{2}$ \\
$d_{\mathrm{E}}(O, P)$ & $\sqrt{M^{2}+y^{2}}$ & $M$ \\
$d_{\mathrm{C}}(O, P)$ & $y b+(M-y) a$ & $y b+\left(\sqrt{M^{2}-y^{2}}-y\right) a$
\end{tabular}

Absolute error: $\mathrm{E}^{\mathrm{abs}}(y)=d_{\mathrm{C}}(O, P)-d_{\mathrm{E}}(O, P)$

$E^{\mathrm{abs}}(y) \quad(b-a) y+M a-\sqrt{M^{2}+y^{2}} \quad(b-a) y+a-\sqrt{M^{2}-y^{2}}-M$

$\frac{\mathrm{d} E^{\mathrm{abs}}}{\mathrm{d} y}\left(y_{1}\right)=0 \quad y_{1}=(b-a) M \sqrt{\frac{1}{1-(b-a)^{2}}} \quad y_{1}=\frac{(b-a) M}{\sqrt{a^{2}+(b-a)^{2}}}$

Absolute error extrema

$E^{\text {abs }}\left(y_{0}\right) \quad(a-1) M$

$E^{\mathrm{abs}}\left(y_{1}\right) \quad\left(a-\sqrt{1-(b-a)^{2}}\right) M$

$(a-1) M$

$E^{\text {abs }}\left(y_{2}\right)$

$(b-\sqrt{2}) M$

$\left(\sqrt{(b-a)^{2}+a^{2}}-1\right) M$

$\left(\frac{b}{\sqrt{2}}-1\right) M$

Relative error: $E^{\mathrm{rel}}(y)=E^{\mathrm{abs}}(y) / d_{\mathrm{E}}(O, P)$

$\frac{\mathrm{d} E^{\mathrm{abs}}}{\mathrm{d} y}\left(y_{1}\right) \quad y_{1}=M \frac{b-a}{a}$

$y_{1}=\frac{M(b-a)}{\sqrt{(b-a)^{2}+a^{2}}}$

$=0$

Relative error extrema

$E^{\text {rel }}\left(y_{0}\right) \quad(a-1)$

$E^{\text {rel }}\left(y_{1}\right) \quad \sqrt{(b-a)^{2}+a^{2}}-1$

$(a-1)$

$E^{\mathrm{rel}}\left(y_{2}\right) \quad \frac{\sqrt{2}}{2}(\sqrt{2}-b)$

$\sqrt{(b-a)^{2}+a^{2}}-1$

$\frac{b}{\sqrt{2}}-1$

computation of optimal coefficients for any mask size or anisotropy factor in 3-D.

\section{Analytical expression of the error with Euclidean distance}

Given a chamfer mask, $m_{\mathrm{C}}=\left\{\left(\mathbf{v}_{i}, \omega_{i}\right)\right\}$, the relative error of the chamfer distance with respect to the Euclidean one depends locally only on a few coefficients. It will be demonstrated how a well chosen decomposition of the mask into regular cones, where the chamfer distance is locally defined, allows to express analytically this local error, and to find its local extrema.

Optimizing over all the local errors results in the computation of the optimal real coefficients $\left\{\omega_{i, \text { opt }}\right\}$ (as conducted in the above section). To the best of our knowledge, this has not been addressed in the general case (i.e. for arbitrary mask size and grid anisotropy) and is beyond the scope of this work. However, knowledge of the error extrema allows to compare different sets of coefficients, and to extract the best sets $\left\{\omega_{i, \text { int }}\right\}$ from all the sets of integer coefficients sorted in lexicographic order. 
In the following, we denote the coordinates of a point $p \in \mathbb{Z}^{3},(x, y, z)$, and the coordinates of a vector $\mathbf{v}_{i} \in \mathbb{Z}^{3},\left(x_{i}, y_{i}, z_{i}\right)$.

\subsection{Mask geometry decomposition}

According to Definition 2.5, a chamfer mask is central-symmetric. This allows considering only one eighth of the space $\mathbb{Z}^{3}$ for the error calculation, this calculation being valid for the whole space thanks to symmetry considerations. In the following, we consider only the first eighth $\frac{1}{8} \mathbb{Z}^{3}$, delimited by the half-lines $(O, \mathbf{x})$, $(O, \mathbf{y})$ and $(O, \mathbf{z})$. The reduction of a chamfer mask $m_{\mathrm{C}}$ to this first eighth will be called a mask generator and denoted $m_{\mathrm{C}}^{\mathrm{g}}$. Fig. 7 shows the generator of the mask of Fig. 2(b).

Estimating the error between a chamfer distance and the Euclidean one can be quite awkward when dealing with large masks. This difficulty can be reduced if we are able to define areas where the chamfer distance is locally defined, that is to say where chamfer distance depends only on few weights of the chamfer mask. To do so, we decompose the chamfer mask into cones, a cone being defined by a triplet of vectors, more exactly into regular cones that exhibit some interesting properties. Such a decomposition is shown in Fig. 8.

Definition 3.1 (Continuous cone)A continuous cone $\left\langle\mathbf{v}_{i}, \mathbf{v}_{j}\right.$, $\left.\mathbf{v}_{k}\right\rangle$ represents the region of $\mathbb{R}^{3}$ delimited by the vectors $\mathbf{v}_{i}, \mathbf{v}_{j}$ and $\mathbf{v}_{k}$. That is:

$$
\begin{aligned}
\left\langle\mathbf{v}_{i}, \mathbf{v}_{j}, \mathbf{v}_{k}\right\rangle & =\{M \in E: \overrightarrow{O M} \\
& \left.=\lambda_{i} \cdot \mathbf{v}_{i}+\lambda_{j} \cdot \mathbf{v}_{j}+\lambda_{k} \cdot \mathbf{v}_{k}, \lambda_{i}, \lambda_{j}, \lambda_{k} \in \mathbb{R}^{+}\right\}
\end{aligned}
$$

Definition 3.2 (Discrete cone) A discrete cone $\left\langle\left\langle\mathbf{v}_{i}, \mathbf{v}_{j}, \mathbf{v}_{k}\right\rangle\right\rangle$ is the set of points in $\mathbb{Z}^{3}$ included in the continuous cone $\left\langle\mathbf{v}_{i}, \mathbf{v}_{j}, \mathbf{v}_{k}\right\rangle$.

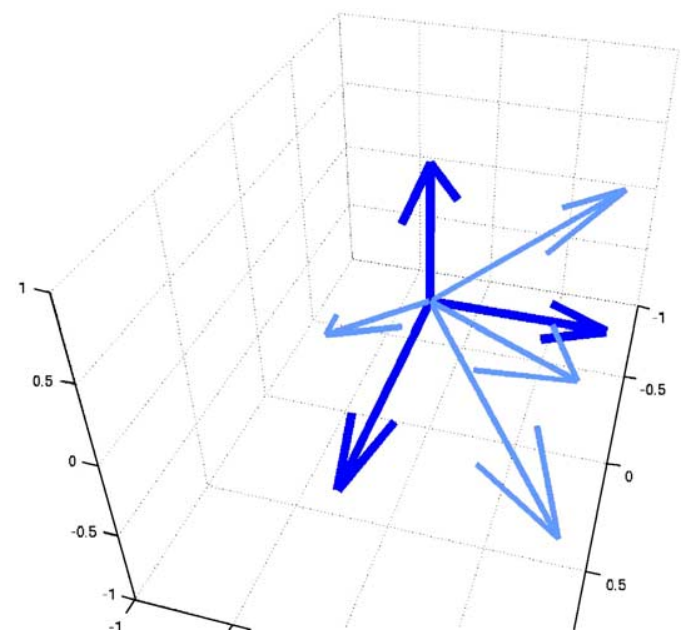

Fig. 7. Reduction of the mask shown in Fig. 2(b) to the first eighth of $\mathbb{Z}^{3}$.

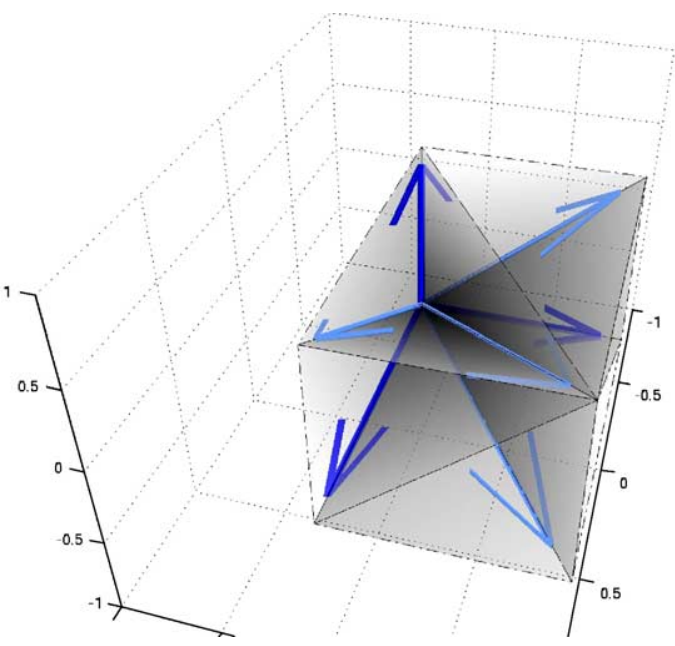

Fig. 8. Mask decomposition into regular cones.

Definition 3.3 (Regular cone) A regular cone is a discrete cone $\left\langle\left\langle\mathbf{v}_{i}, \mathbf{v}_{j}, \mathbf{v}_{k}\right\rangle\right\rangle$ which verifies $\Delta_{i, j, k}= \pm 1$ where

$\Delta_{i, j, k}=\left|\begin{array}{ccc}x_{i} & x_{j} & x_{k} \\ y_{i} & y_{j} & y_{k} \\ z_{i} & z_{j} & z_{k}\end{array}\right|$

A regular cone exhibits the interesting property that any point of such a cone can be reached by an integer linear combination of its three generating vectors $[18,27]$ (see Fig. 9).

Given a point $p(x, y, z)$, finding the coefficients of this linear combination amounts to solving:

$\mathbf{O} p=a \mathbf{v}_{i}+b \mathbf{v}_{j}+c \mathbf{v}_{k}$ i.e. $\left(\begin{array}{l}x \\ y \\ z\end{array}\right)=\left(\begin{array}{ccc}x_{i} & x_{j} & x_{k} \\ y_{i} & y_{j} & y_{k} \\ z_{i} & z_{j} & z_{k}\end{array}\right)\left(\begin{array}{l}a \\ b \\ c\end{array}\right)$

The solution exists if $\Delta_{i, j, k} \neq 0$, i.e. the three vectors $\mathbf{v}_{i}, \mathbf{v}_{j}$, and $\mathbf{v}_{j}$ are independent, and the coefficients of the linear

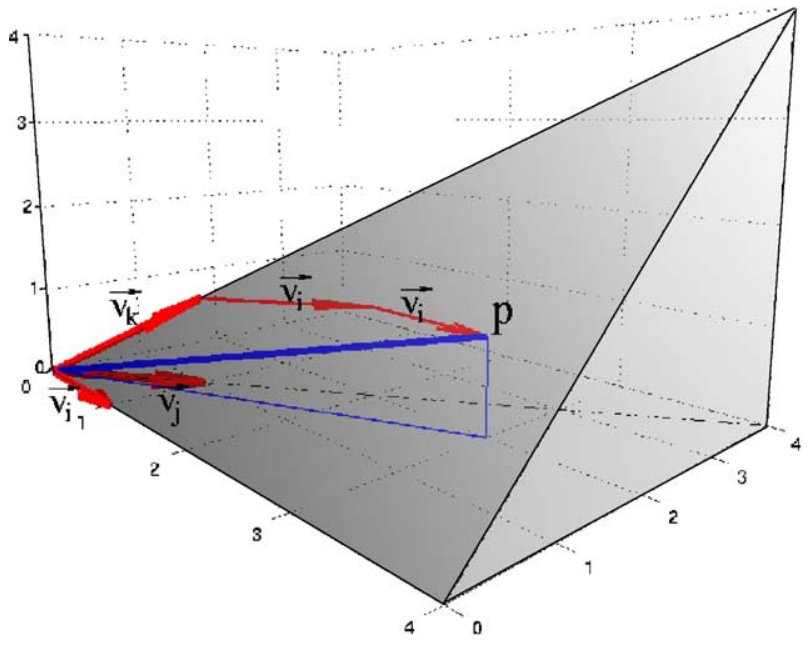

Fig. 9. Sample of regular cone. 
combination are

$a=\frac{1}{\Delta_{i, j, k}}\left|\begin{array}{ccc}x & x_{j} & x_{k} \\ y & y_{j} & y_{k} \\ z & z_{j} & z_{k}\end{array}\right|, \quad b=\frac{1}{\Delta_{i, j, k}}\left|\begin{array}{ccc}x_{i} & x & x_{k} \\ y_{i} & y & y_{k} \\ z_{i} & z & z_{k}\end{array}\right|$,

and $c=\frac{1}{\Delta_{i, j, k}}\left|\begin{array}{ccc}x_{i} & x_{j} & x \\ y_{i} & y_{j} & y \\ z_{i} & z_{j} & z\end{array}\right|$

If the cone $\left\langle\left\langle\mathbf{v}_{i}, \mathbf{v}_{j}, \mathbf{v}_{k}\right\rangle\right\rangle$ is regular, $\Delta_{i, j, k}= \pm 1$. If $p \in \mathbb{Z}^{3}$, then, since all coordinates $\left(x, y, z\right.$, and $x_{l}, y_{l}, z_{l}$, for $\left.l=i, j, k\right)$ are in $\mathbb{Z}$, the three coefficients $a, b, c$ also belong to $\mathbb{Z}$.

The sign of a determinant, e.g.

$\left|\begin{array}{lll}x_{i} & x_{j} & x \\ y_{i} & y_{j} & y \\ z_{i} & z_{j} & z\end{array}\right|$

characterizes half-spaces that are separated here by the plane defined by $\left(O, \mathbf{v}_{i}, \mathbf{v}_{j}\right)$. Since the cone is nothing but the intersection of three half-spaces, it can be shown that $a, b, c$ are positive integers for $p$ belonging to $\left\langle\left\langle\mathbf{v}_{i}, \mathbf{v}_{j}, \mathbf{v}_{k}\right\rangle\right\rangle$.

In the following, we will consider only mask generators decomposed into regular cones, i.e. built with a regular triangulation. It will be shown in Section 5.1 that, this can be easily achieved thanks to Farey series. Moreover, for the sake of simplicity, $\Delta_{i, j, k}=+1$ can also be assumed without loss of generality as this corresponds to a reordering of the vectors $\mathbf{v}_{i}, \mathbf{v}_{j}$, and $\mathbf{v}_{j}$.

\subsection{Analytical expression of the error}

Given a mask generator $m_{\mathrm{C}}^{\mathrm{g}}$ decomposed into regular cones, the error of the chamfer distance with respect to the Euclidean one can be computed. To deal with a more isotropic error distribution, we chose a relative error instead of an absolute one. Given that the chamfer distance is an approximation of the Euclidean distance up to $a$ real multiplicative constant $\varepsilon$ (see Section 2.5 ), the relative error is

$E=\frac{\frac{1}{\varepsilon} d_{\mathrm{C}}-d_{\mathrm{E}}}{d_{\mathrm{E}}}=\frac{d_{\mathrm{C}}}{\varepsilon d_{\mathrm{E}}}-1$

This error will allow the comparison of different sets of coefficients to characterize the best sets to be used in the practical computation of a chamfer distance.

To facilitate this comparison, we will exhibit the extrema of this error, computed on the planes $x=M, y=M$, or $z=M$. It has to be pointed out that the extrema calculation can be conducted independently from a cone to the next, since cones are regular.

Moreover, we will perform these calculations into a 3-D anisotropic cubic lattice where $d_{x}, d_{y}$, and $d_{z}$ are respectively the Euclidean lengths of a voxel in the $\mathbf{x}, \mathbf{y}$, and $\mathbf{z}$ directions.

\subsubsection{Chamfer and Euclidean distances and relative error expressions}

Given a regular cone $\left\langle\left\langle\mathbf{v}_{i}, \mathbf{v}_{j}, \mathbf{v}_{k}\right\rangle\right\rangle$ of the chamfer mask generator $m_{\mathrm{C}}^{\mathrm{g}}$, a point $p(x, y, z)$ of this cone can be expressed as a linear combination of the three vectors $\mathbf{v}_{i}, \mathbf{v}_{j}$, and $\mathbf{v}_{k}$ (Eq. (1)), the three coefficients, $a, b$, and $c$, of this linear combination being given by Eq. (2). By definition, the chamfer distance between $O$ and $p$ is given by

$d_{\mathrm{C}}(O, p)=a \omega_{i}+b \omega_{j}+c \omega_{k}$

Replacing $a, b$, and $c$ by their values (recall that $\Delta_{i, j, k}=1$ ) and reordering the terms allows to express the chamfer distance with respect to $x, y$, and $z$ :

$d_{\mathrm{C}}(O, P)=\alpha x+\beta y+\gamma z$

with

$$
\left\{\begin{array}{c}
\alpha=\left(y_{j} z_{k}-y_{k} z_{j}\right) \omega_{i}+\left(y_{k} z_{i}-y_{i} z_{k}\right) \omega_{j}+\left(y_{i} z_{j}-y_{j} z_{i}\right) \omega_{k} \\
\beta=\left(x_{k} z_{j}-x_{j} z_{k}\right) \omega_{i}+\left(x_{i} z_{k}-x_{k} z_{i}\right) \omega_{j}+\left(x_{j} z_{i}-x_{i} z_{j}\right) \omega_{k} \\
\gamma=\left(x_{j} y_{k}-x_{k} y_{j}\right) \omega_{i}+\left(x_{k} y_{i}-x_{i} y_{k}\right) \omega_{j}+\left(x_{i} y_{j}-x_{j} y_{i}\right) \omega_{k}
\end{array}\right.
$$

The Euclidean distance of a point $p(x, y, z)$ of the anisotropic grid to the origin, is simply expressed by:

$d_{\mathrm{E}}(O, p)=\sqrt{d_{x}^{2} x^{2}+d_{y}^{2} y^{2}+d_{z}^{2} z^{2}}$

The relative error between the chamfer and the Euclidean distances is then

$E=\frac{\alpha x+\beta y+\gamma z}{\varepsilon \sqrt{d_{x}^{2} x^{2}+d_{y}^{2} y^{2}+d_{z}^{2} z^{2}}}-1$

for $p$ belonging to the regular cone $\left\langle\left\langle\mathbf{v}_{i}, \mathbf{v}_{j}, \mathbf{v}_{k}\right\rangle\right\rangle$ and $\alpha, \beta, \gamma$, given by Eq. (5).

\subsubsection{Error computation on a plane}

Depending on the orientation of the cone (see Fig. 8), the error has to be minimized on either the plane $x=M$, or $y=M$ or $z=M$. Without loss of generality, we will only detail the case $x=M(M \neq 0)$. The calculation is conducted in $\mathbb{R}^{3}$. We can then get analytical expressions for the error extrema. The minimum and maximum values of these expressions are respectively minimum and maximum bounds of the error in $\mathbb{Z}^{3}$ : the effective chamfer distance error is then overestimated by this calculation.

To compute the error on the plane $x=M$ we consider points $p(M, y, z)=M\left(1, y^{\prime}, z^{\prime}\right)$ with $y^{\prime}=y / M$ and $z^{\prime}=z / M$ inside the triangle $\left(V_{i}, V_{j}, V_{k}\right)$ where

$V_{l}=\left(M, M \frac{y_{l}}{x_{l}}, M \frac{z_{l}}{x_{l}}\right)$ 


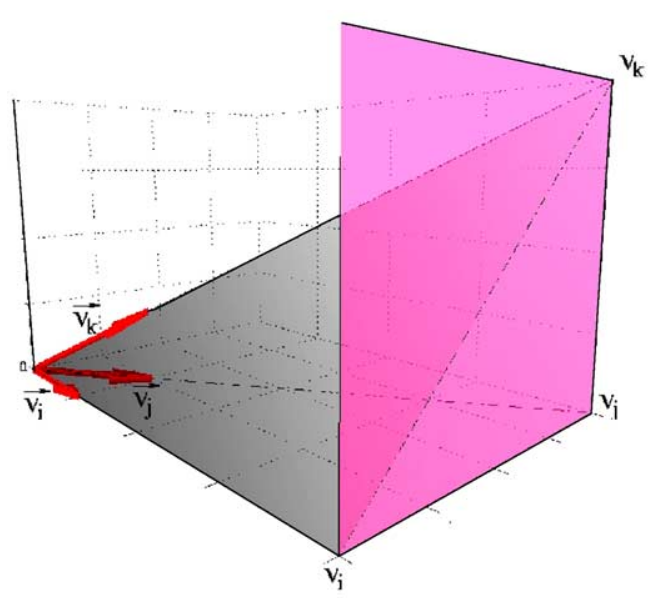

(a)

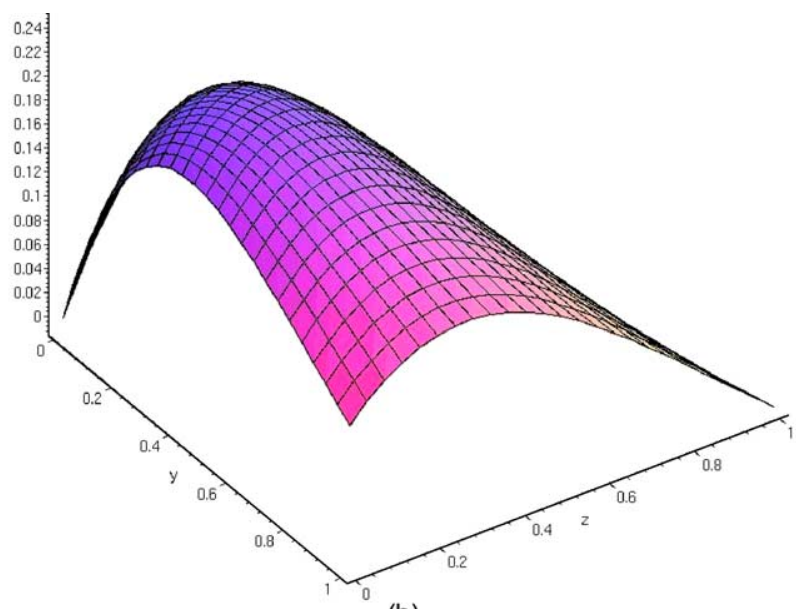

(b)

Fig. 10. Calculation of the error function on the plan $x=M$.

for $l=i, j, k$ (see Fig. 10(a)). On the plane $x=M$, the relative error becomes:

$E_{\text {cone }}(x, y)=\frac{1}{\varepsilon} \frac{\alpha M+\beta y+\gamma z}{\sqrt{d_{x}^{2} M^{2}+d_{y}^{2} y^{2}+d_{z}^{2} z^{2}}}-1$

or

$E_{\text {cone }}\left(x^{\prime}, y^{\prime}\right)=\frac{1}{\varepsilon} \frac{\alpha+\beta y^{\prime}+\gamma z^{\prime}}{\sqrt{d_{x}^{2}+d_{y}^{2} y^{\prime 2}+d_{z}^{2} z^{\prime 2}}}-1$
Those derivatives cancel both at point $p=M\left(1, \beta d_{x}^{2} / \alpha d_{y}^{2}\right.$, $\gamma d_{x}^{2} / \alpha d_{z}^{2}$ ) and the value of this extremum is:

$E_{i j k}^{\mathrm{cone}}=\frac{1}{\varepsilon} \sqrt{\frac{\alpha^{2}}{d_{x}^{2}}+\frac{\beta^{2}}{d_{y}^{2}}+\frac{\gamma^{2}}{d_{z}^{2}}}$

(2) On an edge of the triangle. There are three edges. Without loss of generality, only the calculation for $\left[V_{i} V_{j}\right]$ is presented. A point $p$ belonging to this edge can be represented by $p=r V_{i}+(1-r) V_{j}$, with $0 \leq r \leq 1$, yielding the relative error along the edge:

$$
E_{\text {cone }}(r)=\frac{1}{\varepsilon} \frac{(\beta Y+\gamma Z) r+\left(\alpha+\beta y_{j}+\gamma z_{j}\right)}{\sqrt{\left(d_{y}^{2} Y^{2}+d_{z}^{2} Z^{2}\right) r^{2}+2\left(d_{y}^{2} y_{j} Y+d_{z}^{2} z_{j} Z\right) r+d_{x}^{2}+d_{y}^{2} y_{j}^{2}+d_{z}^{2} z_{j}^{2}}}-1
$$

$E_{\text {cone }}$ is continuous on the compact triangle $\left(V_{i}, V_{j}, V_{k}\right)$. Fig. 10(b) shows such a function for the unique cone of the with $Y=y_{i}-y_{j}$ and $\mathrm{Z}=z_{i}-z_{j}$. The derivative of the above expression cancels for

$$
r_{\max }=-\frac{\left(\beta\left(y_{j} Z-z_{j} Y\right)+\alpha Z\right) z_{j} d_{z}^{2}+\left(\gamma\left(z_{j} Y-y_{j} Z\right)+\alpha Y\right) y_{j} d_{y}^{2}-(\beta Y+\gamma Z) d_{x}^{2}}{\left(\beta\left(y_{j} Z-z_{j} Y\right)+\alpha Z\right) Z d_{z}^{2}+\left(\gamma\left(z_{j} Y-y_{j} Z\right)+\alpha Y\right) Y d_{y}^{2}} .
$$

following mask generator: $m_{\mathrm{C}}^{\mathrm{g}}=\{((1,0,0), 3),((1,1,0), 6)$, $((1,1,1), 8)\}$ in an anisotropic image where $d_{x}=1, d_{y}=1.5$, $d_{z}=2$. As $E_{\text {cone }}$ is continuous on a compact, it is bounded and reaches its bounds. There are three possible locations for its extrema.

(1) Within the triangle. Such an extrema is characterized by $\partial E_{\text {cone }} / \partial y^{\prime}=\partial E_{\text {cone }} / \partial z^{\prime}=0$ with

$\frac{\partial E_{\text {cone }}}{\partial y^{\prime}}\left(y^{\prime}, z^{\prime}\right)=\frac{\beta d_{x}^{2}-\left(\alpha+\gamma z^{\prime}\right) y^{\prime} d_{y}^{2}+\beta z^{\prime 2} d_{z}^{2}}{\varepsilon\left(d_{x}^{2}+d_{y}^{2} y^{\prime 2}+d_{z}^{2} z^{12}\right)^{3 / 2}}$

$\frac{\partial E_{\text {cone }}}{\partial z^{\prime}}\left(y^{\prime}, z^{\prime}\right)=\frac{\gamma d_{x}^{2}-\gamma y \prime^{2} d_{y}^{2}-\left(\alpha+\beta y^{\prime}\right) z^{\prime} d_{z}^{2}}{\varepsilon\left(d_{x}^{2}+d_{y}^{2} y^{\prime 2}+d_{z}^{2} z^{\prime 2}\right)^{3 / 2}}$
$E_{\text {cone }}\left(r_{\text {max }}\right)$, also denoted $E_{i j}^{\text {cone }}$ and whose form is too complicated to be displayed here, is an effective error extremum if $0 \leq r_{\max } \leq 1$. Similar calculations give the expressions of the extremal errors, $E_{i k}^{\text {cone }}$ and $E_{j k}^{\text {cone }}$, along the two others edges.

(3) On a vertex of the triangle. If the extremum of the error occurs on a triangle vertex, it has one of these three values:

$$
\begin{aligned}
& E_{i}^{\text {cone }}=\frac{1}{\varepsilon} \frac{\omega_{i}}{\sqrt{d_{x}^{2} x_{i}^{2}+d_{y}^{2} y_{i}^{2}+d_{z}^{2} z_{i}^{2}}}-1 \\
& E_{j}^{\text {cone }}=\frac{1}{\varepsilon} \frac{\omega_{j}}{\sqrt{d_{x}^{2} x_{j}^{2}+d_{y}^{2} y_{j}^{2}+d_{z}^{2} z_{j}^{2}}}-1
\end{aligned}
$$


$E_{k}^{\mathrm{cone}}=\frac{1}{\varepsilon} \frac{\omega_{k}}{\sqrt{d_{x}^{2} x_{k}^{2}+d_{y}^{2} y_{k}^{2}+d_{z}^{2} z_{k}^{2}}}-1$

Gathering the above results enables the computation of the minimum and maximum values, $\tau_{\min }^{\text {cone }}$ and $\tau_{\max }^{\text {cone }}$, of the relative error for a cone:

$\tau_{\min }^{\text {cone }}=\min \left\{E_{i j k}^{\mathrm{cone}}, E_{i j}^{\mathrm{cone}}, E_{i k}^{\mathrm{cone}}, E_{j k}^{\mathrm{cone}}, E_{i}^{\mathrm{cone}}, E_{j}^{\mathrm{cone}}, E_{k}^{\mathrm{cone}}\right\}$

$\tau_{\max }^{\text {cone }}=\max \left\{E_{i j k}^{\text {cone }}, E_{i j}^{\text {cone }}, E_{i k}^{\text {cone }}, E_{j k}^{\text {cone }}, E_{i}^{\text {cone }}, E_{j}^{\text {cone }}, E_{k}^{\text {cone }}\right\}$

\subsubsection{Centering the error}

The minimum and maximum values of the relative error for a chamfer mask $m_{\mathrm{C}}$ are computed by comparing the minimum and maximum values of this error for all cones of the mask generator:

$\tau_{\min }=\min \left\{\tau_{\min }^{\text {cone }} /\right.$ cone $\left.\in M_{\mathrm{C}}^{\mathrm{g}}\right\} \quad$ and

$\tau_{\max }=\max \left\{\tau_{\max }^{\text {cone }} /\right.$ cone $\left.\in m_{\mathrm{C}}^{\mathrm{g}}\right\}$

We are interested in the maximum error $\tau=\max \left(\left|\tau_{\min }\right|,\left|\tau_{\max }\right|\right)$. To minimize $\tau$, the interval $\left[\tau_{\min }, \tau_{\max }\right]$ have to be centered with respect to zero, so that $\tau_{\max }=-\tau_{\min }$. As shown in the error expressions (6)-(10), $\tau$ depends on the multiplicative constant $\varepsilon$ that acts as an additional degree of freedom. The optimal multiplicative constant $\varepsilon_{\text {opt }}$ that allows to get $\tau_{\max }=-\tau_{\min }$ can be computed by

$\varepsilon_{\mathrm{opt}}=\varepsilon\left(\frac{\tau_{\min }+\tau_{\max }}{2}+1\right)$

as shown in Ref. [28]. Despite the above expression, it does not depend on $\varepsilon$, the original multiplicative constant that serves for the calculation. Indeed, by definition, we have

$\tau_{\text {min }}=\left(\frac{d_{\mathrm{C}}}{\varepsilon d_{\mathrm{E}}}\right)_{\text {min }}-1$ and $\tau_{\text {max }}=\left(\frac{d_{\mathrm{C}}}{\varepsilon d_{\mathrm{E}}}\right)_{\text {max }}-1$

Replacing these values into the $\varepsilon_{\mathrm{opt}}$ expression yields

$$
\begin{aligned}
\varepsilon_{\mathrm{opt}} & =\varepsilon\left(\frac{\left(\frac{d_{\mathrm{C}}}{\varepsilon d_{\mathrm{E}}}\right)_{\text {min }}-1+\left(\frac{d_{\mathrm{C}}}{\varepsilon d_{\mathrm{E}}}\right)_{\text {max }}-1}{2}+1\right) \\
& =\frac{1}{2}\left(\left(\frac{d_{\mathrm{C}}}{d_{\mathrm{E}}}\right)_{\text {min }}+\left(\frac{d_{\mathrm{C}}}{d_{\mathrm{E}}}\right)_{\text {max }}\right)
\end{aligned}
$$

In the following, $\tau_{\text {opt }}$ denotes the optimal maximum relative error obtained with $\varepsilon_{\text {opt }}$ as a multiplicative constant, i.e. $\tau_{\mathrm{opt}}=\tau_{\max }\left(\varepsilon_{\mathrm{opt}}\right)=-\tau_{\min }\left(\varepsilon_{\mathrm{opt}}\right)$.

\section{Local norm criteria}

We are interested in the automated computation of optimal sets of chamfer coefficients. To this end, to check whether a chamfer mask induces a norm or not, the convexity of the equivalent rational balls has to be verified automatically (see Section 2.4). This convexity condition

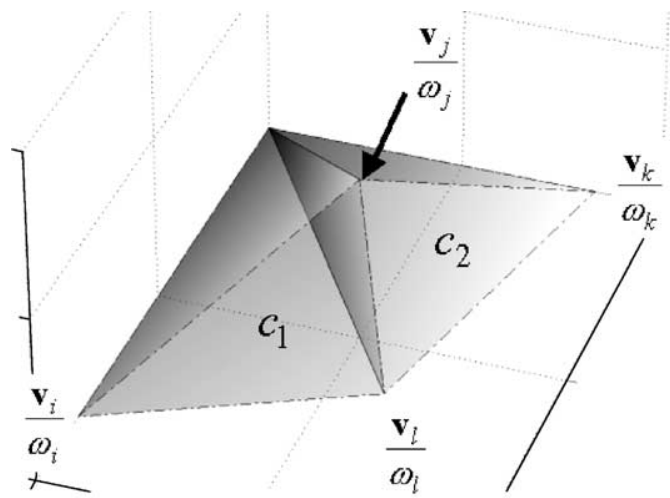

Fig. 11. Geometry of the cone for the local convexity criterion.

means that every edge of an equivalent rational ball must be 'turned inside out'. Let this edge be the common edge of two adjacent cones $c_{1}$ and $c_{2}$, the convexity condition can be analytically expressed by [22]:

$\forall\left(c_{1}, c_{2}\right),\left\{\begin{array}{l}\left.c_{1}=\left\langle\left(\mathbf{v}_{i}, \omega_{i}\right),\left(\mathbf{v}_{j}, \omega_{j}\right),\left(\mathbf{v}_{l}, \omega_{l}\right)\right)\right\rangle \\ \text { and } \\ \left.c_{2}=\left\langle\left(\mathbf{v}_{j}, \omega_{j}\right),\left(\mathbf{v}_{k}, \omega_{k}\right),\left(\mathbf{v}_{l}, \omega_{l}\right)\right\rangle\right\rangle\end{array}, \begin{array}{llll}x_{i} & x_{j} & x_{k} & x_{l} \\ y_{i} & y_{j} & y_{k} & y_{l} \\ z_{i} & z_{j} & z_{k} & z_{l} \\ \omega_{i} & \omega_{j} & \omega_{k} & \omega_{l}\end{array} \mid \geq 0\right.$.

Fig. 11 shows the geometry of a pair of cones for the above local convexity criterion. This edge checking can be limited to the edges of the mask generator. Indeed, for a cone $c_{1}$ of the mask generator, either its neighboring cone $c_{2}$ (a cone sharing an face with $c_{1}$ ) is also in the mask generator (and condition (11) can be tested), or it is outside. In the latter case, symmetry considerations (see Fig. 11) yield $\omega_{k}=\omega_{i}, \mathbf{v}_{k}$ being symmetrical to $\mathbf{v}_{i}$ with respect to plane $\left(O, \mathbf{v}_{j}, \mathbf{v}_{l}\right)$.

\section{Optimal set of coefficients computation}

The computation of the optimal coefficients sets is done by testing the sets of integer coefficients (sorted in lexicographic order) and by keeping those that have an optimal maximum relative error inferior to the one of previously extracted sets. This computation is twofold: first, for the given mask size, we build the corresponding chamfer mask together with its associated regular triangulation; second, when parsing the integer coefficients sets, the local norm conditions are checked and the optimal maximum relative error is computed.

\subsection{Building mask geometry}

The 2 -D $3 \times 3$ and 3 -D $3 \times 3 \times 3$ chamfer masks have already been widely studied in the literature. Dealing with larger 2-D masks is not that difficult since building a regular triangulation is straightforward in 2-D. However, in 3-D, the triangulation of a chamfer mask is not unique, and some of them may be non-regular. In these cases, the error 


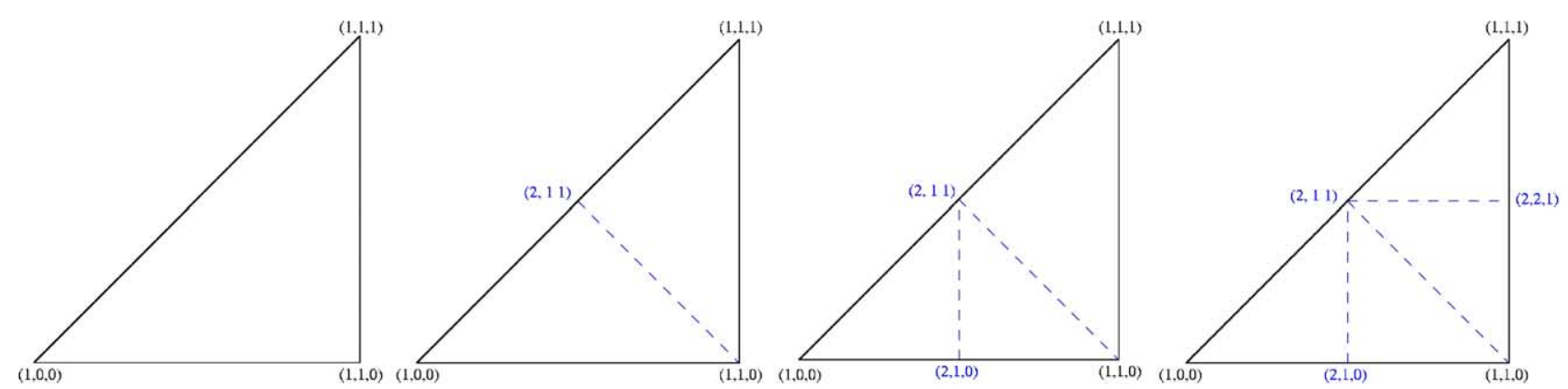

Fig. 12. Construction of $\mathcal{T}_{2}$ from $\mathcal{T}_{1}$. The triangle are projected onto the plane $x=1$. For left to right, the triangulations correspond respectively to masks $3 \times 3 \times 3$ (triangle $\left(\mathbf{V}_{i}, \mathbf{V}_{j}, \mathbf{V}_{k}\right)$ of Fig. $10($ a) $), 3 \times 3 \times 5,3 \times 5 \times 5$, and $5 \times 5 \times 5$.

calculations presented above are not valid any longer. To overcome this problem, Remy [22] introduced rules to obtain regular cones by adding vectors to non-regular ones. But his method imposed to already know the chamfer coefficients at this stage and may fail to give integer coefficients to added vectors. For this reason, we chose to build the chamfer mask together with an associated regular triangulation using Farey series.

\subsubsection{Symmetry considerations}

To reduce computations, we can restrict the triangulation building to the first forty-eighth of space. Indeed, the wellknown $3 \times 3 \times 3$ chamfer mask gives a natural regular triangulation. Its generator (see Fig. 7)

$$
\begin{aligned}
m_{3 \times 3 \times 3}^{\mathrm{g}}= & \left\{\left((1,0,0), \omega_{100}\right),\left((1,1,0), \omega_{110}\right),\left((1,1,1), \omega_{111}\right),\right. \\
& \left((0,1,1), \omega_{011}\right),\left((0,1,0), \omega_{010}\right), \\
& \left.\left((0,0,1), \omega_{001}\right),\left((1,0,1), \omega_{101}\right)\right\}
\end{aligned}
$$

contains six cones which are: the cone $《(1,0,0),(1,1,0)$, $(1,1,1)\rangle\rangle$, its symmetric with respect to the plans $y=z$, their symmetric with respect to the plan $x=y$, and their symmetric with respect to the plane $y=z$ (see Fig. 8). To build larger masks, points have to be added to existing regular cones that have to be divided into new regular cones. Thanks to the above considerations, this have to be done only for the first cone, the rest of the triangulation being deduced by symmetry.

\subsubsection{Farey sets}

A Farey set $\hat{\mathscr{F}}_{n}$ of order $n$ is a set of all the irreducible points $(y / x, z / x)$ in $\mathbb{Q} \cap[0,1]$ whose denominator does not exceed $n$. It turns out that a Farey set contains only visible points.

The Farey set of order $n$ corresponds to the vectors of the generator of a 3 -D chamfer mask of size $(2 n+1)^{3}$. For instance, the lexicographically ordered Farey set of order 1,

$\hat{\mathscr{F}}_{1}=\left\{\left(\frac{0}{1}, \frac{0}{1}\right),\left(\frac{1}{1}, \frac{0}{1}\right),\left(\frac{1}{1}, \frac{1}{1}\right)\right\}$,

corresponds to the set of vectors $\{(1,0,0),(1,1,0),(1,1,1)\}$.

The point is that Farey sets can be built recursively. The Farey set of order $n+1, \hat{\mathcal{F}}_{n+1}$, can be deduced from $\dot{\hat{F}}_{n}$ by

$$
\begin{aligned}
\hat{\mathscr{F}}_{n+1}= & \hat{\mathscr{F}}_{n} \cup\left\{\left(\frac{y}{x}, \frac{z}{x}\right) \hat{+}\left(\frac{y^{\prime}}{x^{\prime}}, \frac{z^{\prime}}{x^{\prime}}\right)\right. \\
& \text { with } \left.x+x^{\prime} \leq n \text { and }\left(\frac{y}{x}, \frac{z}{x}\right), \quad\left(\frac{y^{\prime}}{x^{\prime}}, \frac{z^{\prime}}{x^{\prime}}\right) \in \hat{\mathscr{F}}_{n}\right\}
\end{aligned}
$$

the addition, $\hat{f}$, being defined by [27]

$$
\left(\frac{y}{x}, \frac{z}{x}\right) \hat{+}\left(\frac{y^{\prime}}{x^{\prime}}, \frac{z^{\prime}}{x^{\prime}}\right)=\left(\frac{y+y^{\prime}}{x+x^{\prime}}, \frac{z+z^{\prime}}{x+x^{\prime}}\right) \text {. }
$$

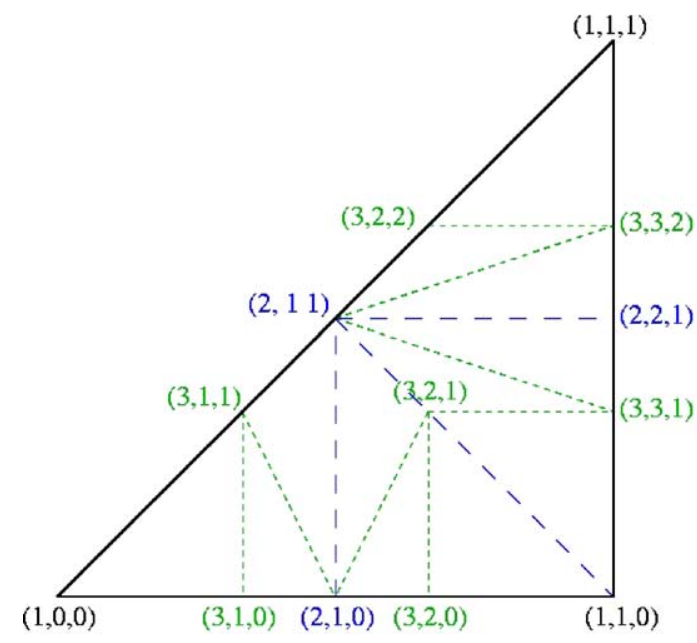

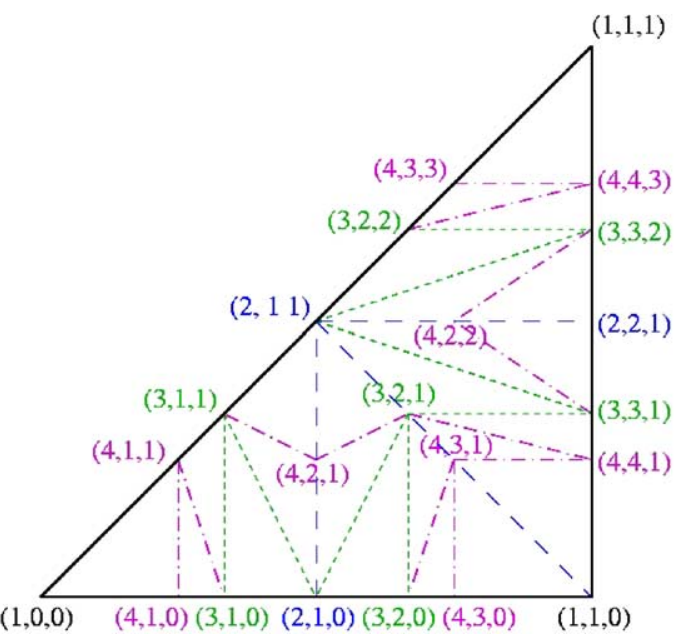

$(1,0,0) \quad(4,1,0)(3,1,0)(2,1,0)(3,2,0)(4,3,0) \quad(1,1,0)$

Fig. 13. $\mathcal{T}_{3}$ and $\mathcal{T}_{4}$. 
Table 2

$3 \times 3 \times 3$ Chamfer mask coefficients

\begin{tabular}{lccccc}
\hline$a$ & $b$ & $c$ & $\varepsilon_{\mathrm{opt}}$ & $\tau_{\mathrm{opt}}(\%)$ & Time $(\mathrm{ms})$ \\
\hline 1 & 1 & 1 & 1.211 & 21.13 & 1 \\
1 & 2 & 2 & 1.207 & 20.71 & 5 \\
2 & 3 & 3 & 2.252 & 12.60 & 5 \\
2 & 3 & 4 & 2.225 & 11.24 & 6 \\
3 & 4 & 5 & 3.244 & 8.14 & 7 \\
4 & 6 & 7 & 4.291 & 7.28 & 9 \\
7 & 10 & 12 & 7.473 & 6.76 & 16 \\
11 & 16 & 19 & 11.740 & 6.73 & 35 \\
12 & 17 & 21 & 12.801 & 6.67 & 52 \\
19 & 27 & 33 & 20.235 & 6.50 & 82 \\
\hline
\end{tabular}

\subsubsection{Recursive construction of regular triangulations}

As discussed in Section 5.1.1, the calculation for the $3 \times 3 \times 3$ chamfer mask can be conducted in the triangulation $\mathcal{T}_{1}$ that consists of one single (and regular) cone, $\langle\langle(1,0,0),(1,1,0),(1,1,1)\rangle)$, or equivalently to the Farey triangle built with point from $\hat{\mathcal{F}}_{1}$,

$$
\left\langle\left\langle\left(\frac{0}{1}, \frac{0}{1}\right),\left(\frac{1}{1}, \frac{0}{1}\right),\left(\frac{1}{1}, \frac{1}{1}\right)\right\rangle\right\rangle .
$$

The vertices of the Farey triangles of the triangulation $\mathcal{T}_{n}$ are the points of the Farey set of order $n$. To build $\mathcal{T}_{n+1}$, the triangles of $\mathcal{T}_{n}$ are split by adding Farey points of order $n+1$ along edges. The construction of $\mathscr{T}_{n+1}$ is achieved when no more Farey points of order $n+1$ can be added. Let us describe more precisely this construction process.

The Farey triangles of $\mathcal{T}_{n}$ are put into a list $\mathcal{L}$. Let us consider a triangle $\langle\langle\mathrm{A}, \mathrm{B}, \mathrm{C}\rangle\rangle$ of $\mathcal{L}$, and its largest edge, ${ }^{2}$ say $A B$. Consider $C^{\prime}=A \hat{+} B$. If $x_{A}+x_{B} \leq n+1, C^{\prime}$ is a point of $\mathcal{F}_{n+1}$, and the triangle $\langle\langle A, B, C\rangle\rangle$ is split into the two triangles $\left\langle\left\langle A, C^{\prime}, C\right\rangle\right\rangle$ and $\left\langle\left\langle C^{\prime}, B, C\right\rangle\right\rangle$. If $\langle\langle A, B, C\rangle\rangle$ is regular, these two triangles are also regular. Indeed, let us consider for instance $\left\langle\left\langle A, C^{\prime}, C\right\rangle\right.$, we, have $\Delta_{A C^{\prime} C}=\Delta_{A(A \hat{+} B) C}=$ $\Delta_{A A C}=\Delta_{A B C}=0+\Delta_{A B C}= \pm 1$. These two triangles, $\left\langle\left\langle A, C^{\prime}, C\right\rangle\right\rangle$ and $\left\langle\left\langle C^{\prime}, B, C\right\rangle\right\rangle$, are put into the list $\mathcal{L}$ for further examination. The construction of $\mathcal{T}_{n+1}$ ends when no more triangles of the list can be split with points of $\mathcal{F}_{n+1}$.

It should be pointed out that the intermediary triangulations obtained when building $\mathcal{T}_{n+1}$ from $\mathcal{T}_{n}$ correspond to intermediary chamfer masks that are regularly triangulated by construction. Fig. 12 details the construction of $\mathcal{T}_{2}$ from $\mathcal{T}_{1}$ and shows such intermediary triangulations, while Fig. 13 displays $\mathcal{T}_{3}$ and $\mathcal{T}_{4}$.

\subsection{Depth-first search algorithm}

Given that we are able to build the regular triangulation of the chamfer mask we are interested in, we can now compute successive integer coefficients $\left\{\omega_{i}\right\}$ with

\footnotetext{
${ }^{2}$ We consider that large discrepancies between the chamfer and the Euclidean distances are more likely to occur along the largest edges.
}

Table 3

$5 \times 5 \times 5$ Chamfer mask coefficients

\begin{tabular}{lcccccccl}
\hline$a$ & $b$ & $c$ & \multicolumn{1}{c}{$d$} & \multicolumn{1}{c}{$f$} & \multicolumn{1}{c}{$\varepsilon_{\text {opt }}$} & $\tau_{\text {opt }}$ & Time \\
\hline 1 & 1 & 1 & 2 & 2 & 2 & 1.211 & 21.13 & $2 \mathrm{~ms}$ \\
1 & 2 & 2 & 3 & 3 & 4 & 1.207 & 20.71 & $8 \mathrm{~ms}$ \\
2 & 2 & 3 & 4 & 4 & 5 & 2.293 & 14.64 & $11 \mathrm{~ms}$ \\
2 & 3 & 3 & 5 & 5 & 6 & 2.252 & 12.60 & $19 \mathrm{~ms}$ \\
2 & 3 & 4 & 5 & 6 & 7 & 2.225 & 11.24 & $20 \mathrm{~ms}$ \\
3 & 4 & 5 & 7 & 7 & 9 & 3.167 & 5.56 & $43 \mathrm{~ms}$ \\
4 & 6 & 7 & 9 & 10 & 13 & 4.179 & 4.49 & $70 \mathrm{~ms}$ \\
5 & 7 & 9 & 11 & 12 & 15 & 5.149 & 2.97 & $80 \mathrm{~ms}$ \\
9 & 13 & 16 & 20 & 22 & 28 & 9.245 & 2.72 & $601 \mathrm{~ms}$ \\
11 & 16 & 20 & 25 & 37 & 34 & 11.288 & 2.62 & $1.5 \mathrm{~s}$ \\
20 & 29 & 35 & 45 & 49 & 62 & 20.5 & 2.5 & $36 \mathrm{~s}$ \\
\hline
\end{tabular}

a decreasing maximum relative error, and, that satisfy norm conditions.

The, first method consists in a brute-force search: all possible sets ${ }^{3}\left\{\omega_{i}\right\}$ are examined in lexicographic order. For a given coefficients set $\left\{\omega_{i}\right\}$, all $\omega_{i}$ being known, we check norm conditions (Eq. (11)) and compute $\tau_{\text {opt }}$ (Section 3.2.3). If this $\tau_{\text {opt }}$ is inferior to the previous computed optimal maximum relative errors, the set $\left\{\omega_{i}\right\}$ is kept as intermediary result.

\subsection{Depth-first search with alpha pruning}

The norm condition (or LNC, see Eq. (11)) check is local and involves only four coefficients. If this check fails for one set $\left\{\omega_{i}\right\}$, it will also fail for the sets $\left\{\omega_{i}^{\prime}\right\}$ that have the same four coefficients. We can then spare computation time by checking those norm conditions as soon as possible (as soon as the four coefficients of two adjacent cones are known), and not when all the coefficients are known.

The search method for optimal coefficients is described by the following pseudo-code

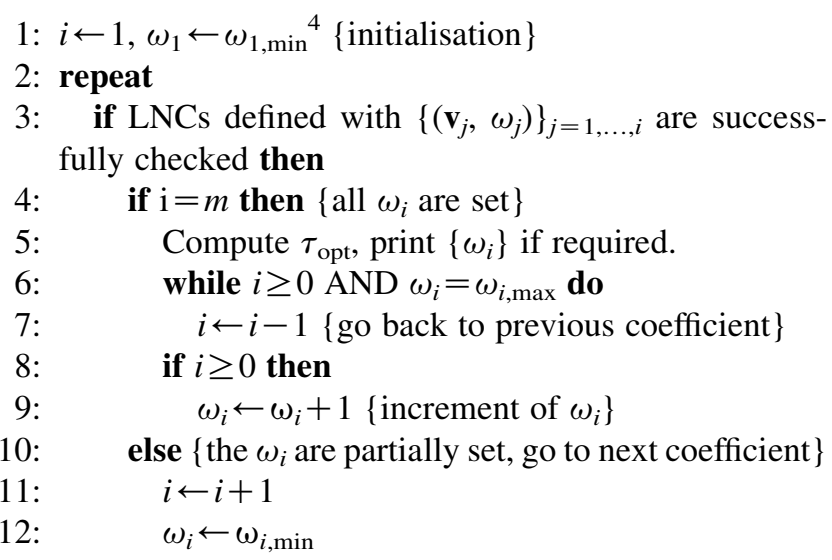

\footnotetext{
${ }^{3}$ Consider that $\omega_{1}$ is associated with vector $\mathbf{x}$. According to that $\omega_{i}$ is fixed, we let $\omega_{i}$ vary from $\omega_{1}\left\|\mathbf{v}_{i}\right\|_{\infty}$ to $\omega_{1}\left\|\mathbf{v}_{i}\right\|_{1}$ : there is then a finite number of sets $\left\{\omega_{i}\right\}$ that are under examination for a fixed $\omega_{1}$.

${ }^{4}$ For the first coefficient, $\omega_{1}$, chosen as the one associated with $\mathbf{x}, \omega_{i, \min }$ and $\omega_{i, \max }$ may be provided by the user. For the other coefficients, $\omega_{i, \min }=$ $\omega_{1}\left\|\mathbf{v}_{i}\right\|_{\infty}$ and $\omega_{i, \min }=\omega_{1}\left\|\mathbf{v}_{i}\right\|_{1}$ (see Section 5.2).
} 
Table 4

$7 \times 7 \times 7$ Chamfer mask coefficients

\begin{tabular}{|c|c|c|c|c|c|c|c|c|c|c|c|c|c|c|c|}
\hline$a$ & $b$ & $c$ & $d$ & $e$ & $f$ & $g$ & $h$ & $i$ & $j$ & $k$ & $l$ & $m$ & $\varepsilon_{\mathrm{opt}}$ & $\tau_{\text {opt }}(\%)$ & Time \\
\hline 1 & 1 & 1 & 2 & 2 & 2 & 3 & 3 & 3 & 3 & 3 & 3 & 3 & 1.211 & 21.13 & $5 \mathrm{~ms}$ \\
\hline 1 & 2 & 2 & 3 & 3 & 4 & 4 & 4 & 5 & 5 & 5 & 6 & 6 & 1.207 & 20.71 & $52 \mathrm{~ms}$ \\
\hline 2 & 2 & 3 & 4 & 4 & 5 & 6 & 6 & 6 & 6 & 7 & 7 & 8 & 2.293 & 14.64 & $87 \mathrm{~ms}$ \\
\hline 2 & 3 & 3 & 5 & 5 & 6 & 7 & 7 & 8 & 8 & 8 & 9 & 9 & 2.252 & 12.60 & $226 \mathrm{~ms}$ \\
\hline 2 & 3 & 4 & 5 & 6 & 7 & 7 & 8 & 8 & 9 & 10 & 10 & 11 & 2.225 & 11.24 & $469 \mathrm{~ms}$ \\
\hline 3 & 4 & 5 & 6 & 7 & 9 & 9 & 9 & 10 & 11 & 12 & 13 & 14 & 3.158 & 5.28 & $5 \mathrm{~s}$ \\
\hline 4 & 6 & 7 & 9 & 10 & 13 & 13 & 14 & 15 & 16 & 17 & 19 & 20 & 4.179 & 4.49 & $29 \mathrm{~s}$ \\
\hline 5 & 7 & 9 & 11 & 12 & 15 & 16 & 16 & 18 & 19 & 21 & 22 & 24 & 5.186 & 3.72 & $1 \min 13 \mathrm{~s}$ \\
\hline 5 & 7 & 9 & 11 & 12 & 15 & 16 & 17 & 18 & 19 & 21 & 22 & 24 & 5.149 & 2.97 & $1 \min 13 \mathrm{~s}$ \\
\hline 7 & 10 & 12 & 16 & 17 & 21 & 22 & 23 & 26 & 27 & 29 & 31 & 33 & 7.176 & 2.51 & $16 \min 3 \mathrm{~s}$ \\
\hline 8 & 11 & 14 & 18 & 19 & 24 & 25 & 26 & 29 & 30 & 33 & 34 & 38 & 8.184 & 2.30 & $44 \min$ \\
\hline 10 & 14 & 17 & 22 & 24 & 30 & 32 & 33 & 36 & 37 & 41 & 43 & 47 & 10.224 & 2.24 & $4 \mathrm{~h} 47 \mathrm{~min}$ \\
\hline 11 & 16 & 19 & 25 & 27 & 34 & 35 & 37 & 41 & 42 & 46 & 49 & 53 & 11.238 & 2.16 & $11 \mathrm{~h}$ \\
\hline 12 & 17 & 21 & 27 & 29 & 36 & 38 & 40 & 44 & 45 & 49 & 52 & 56 & 12.245 & 2.04 & $22 \mathrm{~h}$ \\
\hline 14 & 20 & 24 & 31 & 34 & 43 & 44 & 46 & 51 & 53 & 58 & 62 & 67 & 14.248 & 1.77 & $>24 \mathrm{~h}$ \\
\hline
\end{tabular}

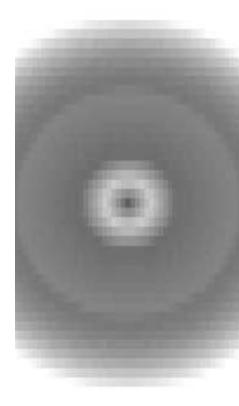

(a)

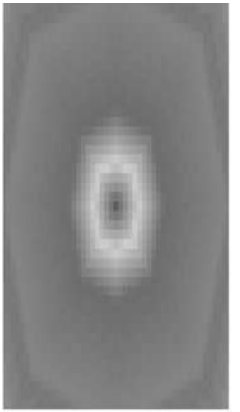

(b)

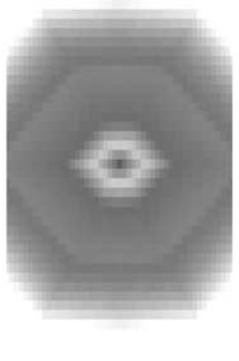

(c)

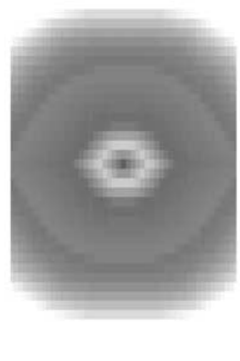

(d)

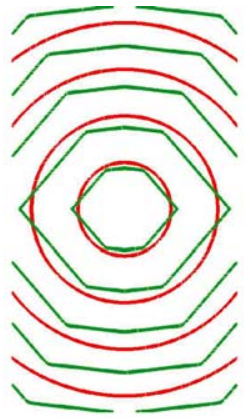

(e)

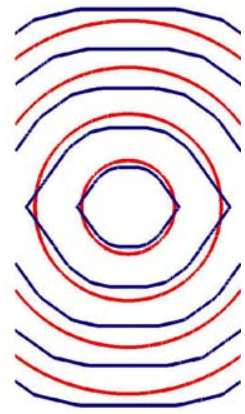

(f)

Fig. 14. Examples of 2-D distance maps on anisotropic grids: (a) Euclidean distance map; (b) chamfer map computed with a $3 \times 3$ mask originally devoted to isotropic images; (c) chamfer map computed with a $3 \times 3$ anisotropic mask whose coefficients where chosen with our method; (d) chamfer map computed with a $5 \times 5$ anisotropic mask whose coefficients where chosen with our method; (e) Euclidean isolines (red) together with $3 \times 3$ anisotropic chamfer map (c) isolines (green); (f) Euclidean isolines (red) together with $5 \times 5$ anisotropic chamfer map (d) isolines (blue) (For interpretation of the references to color in this figure legend, the reader is referred to the web version of this article.).

13: else $\{$ go to next (partial) set $\}$

14: $\quad$ while $i \geq 0$ AND $\omega_{i}=\omega_{i, \max }$ do

15: $\quad i \leftarrow i-1$ \{go back to previous coefficient $\}$

16: $\quad$ if $i \geq 0$ then

17: $\quad \omega_{i} \leftarrow \omega_{i}+1$ increment of $\left.\omega_{i}\right\}$

18: until $i=0$

\section{Results}

We present here some examples of chamfer mask coefficients sets obtained with our method. ${ }^{5}$ This method can be applied to isotropic grids as well as anisotropic grids. For isotropic grids, symmetry allows to reduce

\footnotetext{
${ }^{5}$ A Java implementation is available at http://www.cb.uu.se/ tc18/
}

even more the research area, and to compute larger masks.

\subsection{Masks for isotropic grids}

To simplify notations, we call Farey set points in their lexicographic order: $a(1,0,0), b(1,1,0), c(1,1,1), d(2,1,0)$, $e(2,1,1), f(2,2,1), g(3,1,0), h(3,1,1), i(3,2,0), j(3,2,1)$, $k(3,2,2), l(3,3,1), m(3,3,2)$. We computed coefficient sets, with $\omega_{1}$ varying between 1 and 20 .

Table 2 shows the result for a $3 \times 3 \times 3$ mask (we stop Farey triangulation at the first order), Table 3 shows the result for a $5 \times 5 \times 5$ mask (we stop Farey triangulation at the second order), and Table 4 shows the result for a $7 \times 7 \times 7$ chamfer mask (we stop Farey triangulation to the third order). The last column indicates the time used to find the set of coefficients with our Java code. 
Table 5

$3 \times 3 \times 3$ Chamfer mask coefficients $d_{x}=1, d_{y}=1.2, d_{\mathrm{z}}=2$

\begin{tabular}{|c|c|c|c|c|c|c|c|c|c|}
\hline$a_{X}$ & $a_{Y}$ & $a_{Z}$ & $b_{X Y}$ & $b_{X Z}$ & $b_{Y Z}$ & $c$ & $\varepsilon_{\mathrm{opt}}$ & $\tau_{\mathrm{opt}}(\%)$ & Time \\
\hline 1 & 1 & 2 & 1 & 2 & 2 & 2 & 1.239 & 23.89 & $3 \mathrm{~ms}$ \\
\hline 2 & 2 & 4 & 3 & 4 & 4 & 4 & 2.330 & 16.50 & $9 \mathrm{~ms}$ \\
\hline 3 & 4 & 6 & 5 & 6 & 7 & 7 & 3.486 & 16.20 & $18 \mathrm{~ms}$ \\
\hline 3 & 4 & 6 & 5 & 7 & 7 & 7 & 3.448 & 14.94 & $25 \mathrm{~ms}$ \\
\hline 3 & 4 & 6 & 5 & 7 & 7 & 8 & 3.392 & 13.05 & $26 \mathrm{~ms}$ \\
\hline 4 & 5 & 8 & 6 & 9 & 9 & 10 & 4.474 & 11.86 & $38 \mathrm{~ms}$ \\
\hline 5 & 6 & 10 & 8 & 12 & 12 & 13 & 5.580 & 11.56 & $72 \mathrm{~ms}$ \\
\hline 8 & 10 & 16 & 13 & 18 & 19 & 20 & 8.897 & 11.21 & $178 \mathrm{~ms}$ \\
\hline 9 & 11 & 18 & 14 & 20 & 21 & 23 & 9.973 & 10.81 & $288 \mathrm{~ms}$ \\
\hline 13 & 16 & 26 & 21 & 30 & 31 & 33 & 14.397 & 10.75 & $698 \mathrm{~ms}$ \\
\hline 15 & 18 & 30 & 24 & 34 & 35 & 38 & 16.899 & 10.66 & $1.2 \mathrm{~s}$ \\
\hline 19 & 23 & 38 & 30 & 43 & 45 & 48 & 21.010 & 10.58 & $3 \mathrm{~s}$ \\
\hline
\end{tabular}

Table 6

$3 \times 3 \times 5$ Chamfer mask coefficients $d_{x}=1, d_{y}=1.2, d_{\mathrm{z}}=2$

\begin{tabular}{|c|c|c|c|c|c|c|c|c|c|c|c|c|}
\hline$a_{X}$ & $a_{Y}$ & $a_{Z}$ & $b_{X Y}$ & $b_{X Z}$ & $b_{Y Z}$ & $c$ & $e_{X}$ & $e_{Y}$ & $e_{Z}$ & $\varepsilon_{\mathrm{opt}}$ & $\tau_{\text {opt }}(\%)$ & Time \\
\hline 1 & 1 & 2 & 3 & 4 & 4 & 4 & 6 & 6 & 8 & 1.239 & 23.89 & $4 \mathrm{~ms}$ \\
\hline 2 & 2 & 4 & 3 & 4 & 4 & 4 & 6 & 6 & 8 & 2.330 & 16.50 & $23 \mathrm{~ms}$ \\
\hline 2 & 2 & 4 & 3 & 4 & 4 & 5 & 6 & 7 & 8 & 2.285 & 14.24 & $25 \mathrm{~ms}$ \\
\hline 3 & 4 & 6 & 5 & 7 & 7 & 8 & 10 & 12 & 13 & 3.395 & 13.05 & $57 \mathrm{~ms}$ \\
\hline 4 & 5 & 8 & 6 & 8 & 8 & 9 & 12 & 13 & 16 & 4.521 & 13.03 & $119 \mathrm{~ms}$ \\
\hline 4 & 5 & 8 & 6 & 9 & 9 & 10 & 13 & 15 & 17 & 4.444 & 11.10 & $128 \mathrm{~ms}$ \\
\hline 5 & 6 & 10 & 8 & 11 & 12 & 12 & 16 & 18 & 22 & 5.551 & 11.02 & $306 \mathrm{~ms}$ \\
\hline 5 & 6 & 10 & 8 & 11 & 12 & 13 & 16 & 19 & 22 & 5.491 & 9.81 & $310 \mathrm{~ms}$ \\
\hline 9 & 11 & 18 & 14 & 20 & 21 & 23 & 29 & 34 & 39 & 9.839 & 9.32 & $3 \mathrm{~s}$ \\
\hline 10 & 12 & 20 & 16 & 22 & 23 & 25 & 32 & 37 & 43 & 10.912 & 9.12 & $5 \mathrm{~s}$ \\
\hline 15 & 18 & 30 & 24 & 33 & 35 & 38 & 48 & 56 & 65 & 16.367 & 9.12 & $1 \mathrm{~min}$ \\
\hline 18 & 22 & 36 & 28 & 40 & 12 & 46 & 58 & 67 & 78 & 19.603 & 8.91 & $2 \min 54 \mathrm{~s}$ \\
\hline
\end{tabular}

Table 7

$3 \times 5 \times 5$ Chamfer mask coefficients $d_{x}=1, d_{y}=1.2, d_{z}=2$

\begin{tabular}{|c|c|c|c|c|c|c|c|c|c|c|c|c|c|c|c|c|c|c|}
\hline$a_{X}$ & $a_{Y}$ & $a_{Z}$ & $b_{X Y}$ & $b_{X Z}$ & $b_{Y Z}$ & $c$ & $e_{X}$ & $e_{Y}$ & $e_{Z}$ & $d_{X Y}$ & $d_{X Z}$ & $d_{Y X}$ & $d_{Y Z}$ & $d_{Z X}$ & $d_{Z Y}$ & $\varepsilon_{\mathrm{opt}}$ & $\begin{array}{l}\tau_{\mathrm{opt}} \\
(\%)\end{array}$ & Time \\
\hline 1 & 1 & 2 & 1 & 2 & 2 & 2 & 2 & 3 & 4 & 2 & 2 & 2 & 3 & 4 & 4 & 1.180 & 17.99 & $24 \mathrm{~ms}$ \\
\hline 2 & 2 & 4 & 3 & 4 & 4 & 4 & 5 & 6 & 8 & 4 & 5 & 5 & 6 & 8 & 8 & 2.243 & 12.13 & $48 \mathrm{~ms}$ \\
\hline 4 & 5 & 8 & 6 & 8 & 8 & 9 & 11 & 13 & 16 & 8 & 11 & 11 & 12 & 16 & 16 & 4.474 & 11.842 & $784 \mathrm{~ms}$ \\
\hline 4 & 5 & 8 & 6 & 10 & 9 & 10 & 12 & 15 & 18 & 9 & 12 & 11 & 14 & 18 & 17 & 4.444 & 11.10 & $868 \mathrm{~ms}$ \\
\hline 5 & 6 & 10 & 7 & 11 & 10 & 11 & 13 & 16 & 20 & 10 & 13 & 13 & 15 & 20 & 20 & 5.540 & 10.79 & $2 \mathrm{~s}$ \\
\hline 5 & 6 & 10 & 7 & 10 & 10 & 11 & 14 & 16 & 20 & 11 & 13 & 13 & 15 & 20 & 20 & 5.511 & 10.23 & $2 \mathrm{~s}$ \\
\hline 5 & 6 & 10 & 8 & 11 & 12 & 13 & 16 & 19 & 22 & 12 & 14 & 14 & 18 & 21 & 21 & 5.509 & 10.17 & $3 \mathrm{~s}$ \\
\hline 5 & 6 & 10 & 8 & 12 & 12 & 13 & 16 & 19 & 22 & 12 & 15 & 14 & 18 & 21 & 21 & 5.468 & 9.37 & $3 \mathrm{~s}$ \\
\hline 6 & 7 & 12 & 9 & 13 & 14 & 15 & 18 & 22 & 25 & 14 & 17 & 16 & 21 & 25 & 24 & 6.550 & 9.46 & $9 \mathrm{~s}$ \\
\hline 6 & 7 & 12 & 9 & 13 & 14 & 15 & 18 & 22 & 25 & 14 & 17 & 16 & 21 & 24 & 25 & 6.542 & 9.04 & $9 \mathrm{~s}$ \\
\hline 7 & 8 & 14 & 10 & 15 & 15 & 16 & 20 & 24 & 28 & 15 & 19 & 18 & 23 & 28 & 28 & 7.631 & 9.01 & $21 \mathrm{~s}$ \\
\hline 7 & 8 & 14 & 11 & 15 & 16 & 17 & 21 & 25 & 29 & 16 & 19 & 19 & 24 & 29 & 28 & 7.617 & 8.81 & $26 \mathrm{~s}$ \\
\hline 10 & 12 & 20 & 15 & 21 & 22 & 24 & 29 & 35 & 41 & 22 & 27 & 26 & 33 & 41 & 40 & 10.877 & 8.77 & $34 \mathrm{~s}$ \\
\hline 10 & 12 & 20 & 15 & 24 & 22 & 24 & 29 & 35 & 43 & 22 & 29 & 26 & 33 & 43 & 41 & 10.856 & 8.56 & $34 \mathrm{~s}$ \\
\hline 11 & 13 & 22 & 16 & 23 & 24 & 26 & 32 & 38 & 44 & 24 & 30 & 28 & 36 & 44 & 44 & 11.923 & 8.39 & $14 \min 55 \mathrm{~s}$ \\
\hline 11 & 13 & 22 & 17 & 23 & 24 & 26 & 32 & 38 & 44 & 24 & 30 & 29 & 36 & 44 & 44 & 11.922 & 8.38 & $16 \min 4 \mathrm{~s}$ \\
\hline 12 & 14 & 24 & 18 & 25 & 26 & 28 & 34 & 41 & 48 & 26 & 32 & 31 & 39 & 48 & 48 & 13.001 & 8.34 & $29 \min 9 \mathrm{~s}$ \\
\hline 15 & 18 & 30 & 23 & 33 & 34 & 37 & 45 & 54 & 63 & 34 & 42 & 40 & 51 & 62 & 61 & 16.223 & 8.15 & $4 \mathrm{~h} 23 \mathrm{~min}$ \\
\hline 16 & 19 & 32 & 24 & 35 & 36 & 39 & 48 & 57 & 66 & 36 & 44 & 42 & 54 & 65 & 65 & 17.302 & 8.14 & $7 \mathrm{~h} 23 \mathrm{~min}$ \\
\hline 16 & 19 & 32 & 25 & 35 & 36 & 39 & 48 & 57 & 66 & 36 & 44 & 43 & 54 & 65 & 65 & 17.301 & 8.13 & $7 \mathrm{~h} 44 \mathrm{~min}$ \\
\hline 17 & 20 & 34 & 26 & 37 & 38 & 41 & 50 & 60 & 70 & 38 & 46 & 45 & 57 & 70 & 68 & 18.379 & 8.11 & $12 \mathrm{~h} 17 \mathrm{~min}$ \\
\hline
\end{tabular}


Table 8

$3 \times 3 \times 3$ Chamfer mask coefficients for $d_{x}=1, d_{y}=1, d_{z}=1.5$

\begin{tabular}{lccccccc}
\hline$a_{X}=a_{Y}$ & $b_{X Y}$ & $a_{Z}$ & $b_{X Z}=b_{Y Z}$ & \multicolumn{1}{c}{$c$} & \multicolumn{1}{c}{$\varepsilon_{\mathrm{opt}}$} & $\tau_{\mathrm{opt}}(\%)$ & Time \\
\hline 1 & 1 & 2 & 2 & 2 & 1.313 & 31.31 & $3 \mathrm{~ms}$ \\
1 & 2 & 2 & 2 & 2 & 1.222 & 22.20 & $6 \mathrm{~ms}$ \\
2 & 2 & 3 & 3 & 3 & 2.347 & 17.35 & $8 \mathrm{~ms}$ \\
$\mathbf{2}$ & $\mathbf{3}$ & $\mathbf{3}$ & $\mathbf{4}$ & $\mathbf{4}$ & 2.232 & 11.59 & $12 \mathrm{~ms}$ \\
4 & 6 & 6 & 7 & 8 & 4.393 & 9.83 & $36 \mathrm{~ms}$ \\
$\mathbf{5}$ & $\mathbf{7}$ & $\mathbf{8}$ & $\mathbf{9}$ & $\mathbf{1 0}$ & 5.452 & 9.04 & $62 \mathrm{~ms}$ \\
8 & 11 & 12 & 14 & 16 & 8.705 & 8.81 & $234 \mathrm{~ms}$ \\
12 & 17 & 18 & 22 & 25 & 13.026 & 8.57 & $488 \mathrm{~ms}$ \\
16 & 23 & 24 & 29 & 33 & 17.347 & 8.42 & $1.28 \mathrm{~s}$ \\
17 & 24 & 26 & 31 & 35 & 18.176 & 8.33 & $3 \mathrm{~s}$ \\
\hline
\end{tabular}

Coefficients are ordered as in Ref. [8]. Sets in bold have also been found by Sintorn.

These tables allow to choose a trade-off between precision and computational time when computing chamfer distances. Indeed, if we use Rosenfeld's algorithm,

- with a $3 \times 3 \times 3$ mask, the algorithm performs 13 operations on each pixel and the maximum error is about $6.5 \%$,

- with a $5 \times 5 \times 5$ mask, the algorithm performs 37 operations on each pixel and the maximum error is about $2.5 \%$,

- with a $7 \times 7 \times 7$ mask, the algorithm performs 97 operations on each pixel and the maximum error is less than $2 \%$.

\subsection{Anisotropic masks}

\subsubsection{2-D distance maps}

Fig. 14 shows a distance computed from the center point of an anisotropic 2-D image $\left(d_{x}=1\right.$ and $\left.d_{y}=2\right)$.

\subsubsection{3-D chamfer masks}

Here, we present results for 3-D chamfer masks computed in an anisotropic grid with $d_{x}=1.0, d_{y}=1.2$ and $d_{z}=2.0$. The notations of coefficients as are follows: $a_{X}(1,0,0), a_{Y}(0,1,0), a_{Z}(0,0,1), \quad b_{X Y}(1,1,0), \quad b_{X Z}(1,0,1)$, $b_{Y Z}(0,1,1), c(1,1,1), d_{X Y}(2,1,0), d_{X Z}(2,0,1), d_{Y X}(1,2,0)$, $d_{Y Z}(0,2,1), \quad d_{Z X}(1,0,2), d_{Z Y}(0,1,2), \quad e_{X}(2,1,1), \quad e_{Y}(1,2,1)$, $e_{Z}(1,1,2), f_{X Y}(2,2,1), f_{X Z}(2,1,2), f_{Y Z}(1,2,2)$.

Table 5 shows the result for a $3 \times 3 \times 3$ chamfer mask. Table 6 shows the result for a $3 \times 3 \times 5$ mask, which means that we stopped the Farey triangulation at the first new point of the second order. Table 7 shows the result for a $3 \times 5 \times 5$ mask.

Our results can also be compared with coefficients found in Ref. [8]. Table 8 presents coefficients obtained for $d_{x}=d_{y}=1$ and $d_{z}=1.5$.

\section{Conclusion}

An automated approach to compute optimal chamfer norm coefficients for mask of arbitrary size and for lattice of arbitrary anisotropy has been described. It was enabled by the automated construction of the chamfer mask together with its regular triangulation, this step being based on Farey series. The decomposition of the chamfer mask into regular cones allowed us to derive analytical expressions of the error extrema. By accumulating the error extrema values for all the triangle, calculating the maximum relative error for a set of chamfer coefficients became straightforward. The above error calculation, together with the automated mask construction, was used to compute optimal chamfer coefficients for different mask sizes and grid anisotropies. In addition, these coefficient sets satisfy norm constraints, and thus yield scale invariant chamfer maps.

\section{References}

[1] C. Pudney, Distance-ordered homotopic thinning: a skeletonization algorithm for 3D digital images, Computer Vision and Image Understanding 72 (3) (1998) 404-413.

[2] G. Herman, J. Zheng, C. Bucholtz, Shape-based interpolation, IEEE Computer Graphics and Applications 15 (6) 1992; 69-79.

[3] R. Turner, How much cortex can a vein drain? Downstream dilution of activation-related cerebral blood oxygenation changes, NeuroImage 16 (4) (2002) 1062-1067.

[4] H.M. Duvernoy, S. Selon, J.L. Vannson, Cortical blood vessels of the human brain, Brain Research Bulletin 7 (5) 1981; 519-579.

[5] C. Fouard, G. Malandain, S. Prohaska, M. Westerhoff, F. Cassot, C. Mazel, D. Asselot, J. Marc-Vergnes, Skeletonization by blocks for large 3D datasets: application to brain microcirculation, International Symposium on Biomedical Imaging: From Nano to Macro (ISBI'04), IEEE 2004; 89-92.

[6] O. Cuisenaire, Distance transformations: fast algorithm and applications to medical image processing, $\mathrm{PhD}$ thesis, Université Catholique de Louvain, October 1999.

[7] G. Borgefors, On digital distance transforms in three dimensions, Computer Vision and Image Understanding 64 (3) (1996) 368-376.

[8] I.-M. Sintorn, G. Borgefors, Weighted distance transforms for volume images digitized in elongated voxel grids, Pattern Recognition Letters 25 (5) (2004) 571-580.

[9] E. Remy, E. Thiel, Optimizing 3D chamfer masks with norm constraints, International Workshop on Combinatorial Image Analysis $2000 ; 39-56$.

[10] E. Theil, Géométrie des distances de chanfrein, Habilitation à Diriger des Recherches 2001; Université de la méditerranée (Aix-Marseille 11).

[11] F. Shih, O. Mitchell, A mathematical morphology approach to Euclidean distance transformation, IEEE Transactions on Image Processing 1 (2) (1992) 197-204.

[12] C. Huang, O. Mitchell, A Euclidean distance transform using grayscale morphology decomposition, IEEE Transactions on Pattern Analysis and Machine Intelligence 16 (4) (1994) 443-448.

[13] T. Saito, J.-I. Toriwaki, New algorithms for Euclidean distance transformation of an $n$-dimensional digitized picture with applications, Pattern Recognition 27 (11) (1994) 1551-1565.

[14] T. Hirata, A unified linear-time algorithm for computing distance maps, Information Processing Letters 58 (1996) 129-133.

[15] P. Danielsson, Euclidean distance mapping, Computer Graphics and Image Processing 14 (1980) 227-248.

[16] I. Ragnemalm, The Euclidean distance transform in arbitrary dimensions, Pattern Recognition Letters 14 (11) (1993) 883-888.

[17] D. Coeurjolly, Algorithmique et géométrie discréte pour la caractérisation des courbes et des surfaces, $\mathrm{PhD}$ thesis, Université Lumiére Lyon 2, Bron, Laboratoire ERIC, December 2002. 
[18] U. Montanari, A method for obtaining skeletons using a quasiEuclidean distance, Journal of the Association for Computing Machinery 15 (1968) 600-624.

[19] G. Borgefors, Distance transformations in arbitrary dimensions, Computer Vision, Graphics, and Image Processing 27 (1984) 321-345.

[20] A. Rosenfeld, J. Pfaltz, Sequential operations in digital picture processing, Journal of the Association for Computing Machinery 13 (4) (1966) 471-494.

[21] B. Verwer, Distance transforms: metrics, algorithms and applications, $\mathrm{PhD}$ thesis, Technische Universiteit, 1991.

[22] E. Remy, Normes de chanfrein et axe médian dans le volume discret, $\mathrm{PhD}$ thesis, Université de la Méditerranée, Marseille, France, December 2001.
[23] G. Borgefors, Distance transformations in digital images, Computer Vision, Graphics, and Image Processing 34 (3) (1986) 344-371.

[24] B. Verwer, Local distances for distance transformations in two and three dimensions, Pattern Recognition Letters 12 (1991) 671-682.

[25] D. Coquin, P. Bolon, Discrete distance operator on rectangular grids, Pattern Recognition Letters 16 (1995) 911-923.

[26] J. Mangin, I. Bloch, J. López-Krahe, V. Frouin, Chamfer distances in anisotropic 3D images, VII European Signal Processing Conference, Edinburgh, UK 1994; 975-978.

[27] G. Hardy, E. Wright, An Introduction to the Theory of Numbers, 5th ed, Clarendon Press, Oxford, 1979.

[28] E. Thiel, Les distances de chanfrein en analyse d'images: fondements et applications, PhD thesis, Université Joseph Fourier, September 1994. 